\title{
Erratum
}

\section{2-D VERSUS 3-D MAGNETOTELLURIC DATA INTERPRETATION}

\author{
JUANJO LEDO
}

In the above-mentioned paper, which was published in Volume 26, No. 5 on pp. 511-543, a few author's corrections in the text were not incorporated.

Furthermore, Figures 5 to 13 were displayed incorrectly and the wrong figures were positioned above the captions.

We sincerely regret these errors and would like to make sure the correct information is published to increase the scientific value of the paper.

On the following pages you will find the corrected article, as approved by the author. 


\title{
2-D VERSUS 3-D MAGNETOTELLURIC DATA INTERPRETATION
}

\author{
JUANJO LEDO ${ }^{1,2}$ \\ ${ }^{1}$ Present Geological Survey of Canada, 615 Booth St., Ottawa K1A 0E9, ON, Canada \\ ${ }^{2}$ Departament de Geodinàmica i Geofísica, Universitat de Barcelona, Martí i Franques $s / n$, \\ 08028, Barcelona Spain \\ (E-mail: jledo@ub.edu)
}

(Received 30 August 2002; accepted 30 March 2004)

\begin{abstract}
In recent years, the number of publications dealing with the mathematical and physical 3-D aspects of the magnetotelluric method has increased drastically. However, field experiments on a grid are often impractical and surveys are frequently restricted to single or widely separated profiles. So, in many cases we find ourselves with the following question: is the applicability of the 2-D hypothesis valid to extract geoelectric and geological information from real 3-D environments? The aim of this paper is to explore a few instructive but general situations to understand the basics of a 2-D interpretation of 3-D magnetotelluric data and to determine which data subset (TE-mode or TM-mode) is best for obtaining the electrical conductivity distribution of the subsurface using 2-D techniques. A review of the mathematical and physical fundamentals of the electromagnetic fields generated by a simple 3-D structure allows us to prioritise the choice of modes in a 2-D interpretation of responses influenced by 3-D structures. This analysis is corroborated by numerical results from synthetic models and by real data acquired by other authors. One important result of this analysis is that the mode most unaffected by 3-D effects depends on the position of the 3-D structure with respect to the regional 2-D strike direction. When the 3-D body is normal to the regional strike, the TE-mode is affected mainly by galvanic effects, while the TM-mode is affected by galvanic and inductive effects. In this case, a 2-D interpretation of the TM-mode is prone to error. When the 3-D body is parallel to the regional 2-D strike the TE-mode is affected by galvanic and inductive effects and the TM-mode is affected mainly by galvanic effects, making it more suitable for 2-D interpretation. In general, a wise 2-D interpretation of 3-D magnetotelluric data can be a guide to a reasonable geological interpretation.
\end{abstract}

Keywords: 2-D modeling, 3-D modeling, magnetotelluric theory

\section{Introduction}

In recent years, the electromagnetic geophysical community has developed faster and more reliable codes to calculate the forward electromagnetic response of three-dimensional (3-D) Earth models. Consequently, the current state-of-the-art for magnetotelluric (MT) data interpretation is 3-D 
trial-and-error forward model fitting that is being used more frequently for hypothesis testing, and routine 3-D inversions are on the horizon (Mackie and Madden, 1993; Alumbaugh and Newman, 2000; Newman and Alumbaugh, 2000; Zhdanov et al. 2000; Sasaki, 2001; Newman et al., 2002; Siripunvaraporn et al., 2005). Data acquisition on highly dense 2-D grids has been undertaken to study geothermal (e.g., Park and Torres-Verdin, 1988; Takasugi et al., 1992) and mining-scale problems (e.g., Zhang et al., 1998; Tournerie et al. 2000), but regional-scale field experiments on a 2-D grid are often impractical due to high cost and inaccessibility. Accordingly, regional scale surveys are often restricted to a single profile or widely-separated profiles. The use of additional geophysical and geological information may allow 3-D modelling of MT data (Unsworth et al., 1999; Ledo et al., 2000; Park and Mackie, 2000; Simpson, 2000; Hoffmann-Rothe et al., 2001; Monteiro-Santos et al., 2002) even where the data were collected along a linear profile. Depending on the inductive and geological length scales of the target, 2-D interpretation of the data may be appropriate for a limited number of sites and over a limited period band. Thus, interpretation of magnetotelluric (MT) data for three-dimensional (3-D) regional conductivity structures remains uncommon, and two-dimensional (2-D) models are often considered as an adequate or necessary approach.

The problems and limitations of 2-D magnetotelluric interpretation of 3-D data have been a recurrent topic since the development of the first numerical codes to solve the multi-dimensional magnetotelluric problem (Weidelt, 1975; Jones and Vozoff, 1978; Kaufman and Keller, 1981; Ting and Hohmann, 1981; Hermance, 1982; Jones, 1983; Park et al., 1983; Wannamaker et al., 1984a, b, 1991; Berdichevsky et al., 1998; Berdichevsky, 1999; Wannamaker, 1999). Additionally, several other authors have addressed some specific points (i.e. Park, 1985; Szarka et al., 1994; de Lugao and Kriegshauser, 1997; Spichak et al., 1999, Spichak, 1999; Dosso and Chen, 2000; Osella et al., 2000; Ádám and Kis, 2001; Ledo et al., 2002a). In this review, we consider two main situations for the interpretation of 3-D magnetotelluric data using 2-D techniques. The first situation consists of a 2-D regional-scale structure with the presence of medium-scale 3-D bodies. The 3-D structures that we are considering here are large enough that their effect on the regional data cannot be removed by classic galvanic decomposition techniques. In this case, we want to know which parts of the magnetotelluric data set are more affected by the presence of 3-D structures, and what we have to do to obtain the most "realistic" 2-D model unaffected by the 3-D structures. The 3-D bodies in this case could be considered as geological noise. The second case is the study of 3$\mathrm{D}$ medium-scale bodies (e.g., mining or environmental scale problems) with the use of 2-D techniques; in this case, the 3-D bodies are the targets.

In this paper, emphasis is placed on a few instructive but general situations to understand the basics of the 2-D interpretation of 3-D magnetotelluric data 
within the two scenarios described previously. We show that 2-D geophysical interpretation of 3-D data can nevertheless be a guide to a reasonable geological interpretation in many cases. This review does not pretend to give a set of generic rules valid for all possible cases and specific situations that can be found in the application of the magnetotelluric method to real geological problems.

We start with a review of the mathematical and physical fundamentals of the electromagnetic fields generated by $3-\mathrm{D}$ structures. This is followed by the analysis of several cases of the interpretation of quasi 2-D data. Then the interpretation of a 3-D isolated conductive structure with 2-D techniques is presented in detail.

\section{Mathematical and Physical Fundamentals}

In this section, we develop the mathematical and physical fundamentals to understand the behavior of, and to characterise the fields generated by, the presence of a 3-D body. We establish the relationships between the regional 2-D electromagnetic fields, the scattered fields and the measured fields. We show that it is not possible to obtain an analytical expression for the scattered fields, although we use some approximations to obtain an expression that help us to understand the different effects from the presence of a 3D body on the measured fields.

The following discussion is based on several papers dealing with the solution of Maxwell's equations to calculate the magnetotelluric response of three-dimensional structures (Harrington, 1961; Hohmann, 1975; Ting and Hohmann, 1981; Wannamaker et al., 1984a, b; Habashy et al., 1993; Chave and Smith, 1994; Berdichevsky and Dmitriev, 1997).

We consider a 3-D body within a regional 2-D medium (Figure 1), in which the scattered fields $\mathbf{E}^{\mathrm{s}}$ and $\mathbf{H}^{\mathrm{s}}$ due to the 3-D body can be expressed as:

$$
\mathbf{E}^{\mathrm{s}}=-i \omega \mu \mathbf{A}+\frac{1}{i \omega \varepsilon} \nabla(\nabla \cdot \mathbf{A})
$$

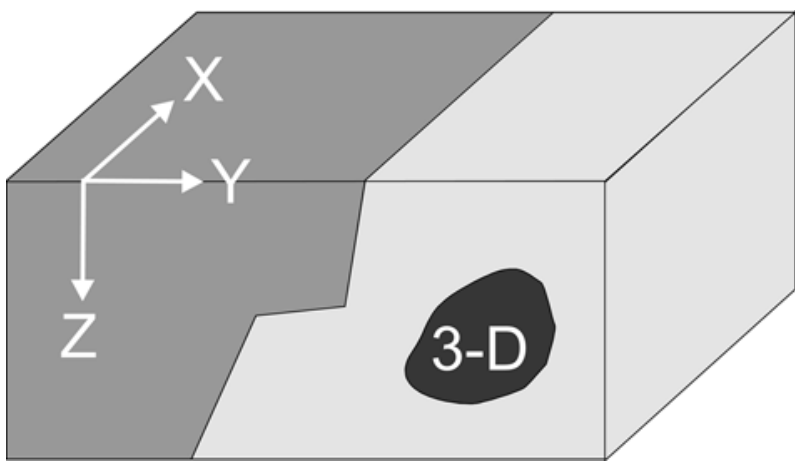

Figure 1. A simple 3-D heterogeneity embedded in a regional 2-D model. 


$$
\mathbf{H}^{\mathrm{s}}=-\nabla \times \mathbf{A}
$$

where $\mathbf{A}$ is the magnetic vector potential (see Appendix), $\omega$ is the angular frequency, $\mu$ is the magnetic permeability and $\varepsilon$ the electric permittivity. The first term on the right side of Equation 1 is associated with the volume currents inside the body and the second term is associated with charge accumulation at the conductivity discontinuities.

Suppose now that an electromagnetic wave propagating in the $x$ direction parallel to the strike direction is incident on the 2-D regional structure (Figure 1). In this case, the vector potential $\mathbf{A}=\left(\mathrm{A}_{x}, 0,0\right)$ only has an $x$-component. Expanding equations 1 and 2 we can arrive at the following expressions:

$$
\begin{aligned}
& E_{x}^{\mathrm{s}}=-i \omega \mu A_{x}+\frac{1}{i \omega \varepsilon} \frac{\partial^{2} A_{x}}{\partial x^{2}} \quad H_{x}^{\mathrm{s}}=0 \\
& E_{y}^{\mathrm{s}}=\frac{1}{i \omega \varepsilon} \frac{\partial^{2} A_{x}}{\partial y \partial x} \quad H_{y}^{\mathrm{s}}=-\frac{\partial A_{x}}{\partial z} \\
& E_{z}^{\mathrm{s}}=\frac{1}{i \omega \varepsilon} \frac{\partial^{2} A_{x}}{\partial z \partial x} \quad H_{z}^{\mathrm{s}}=-\frac{\partial A_{x}}{\partial y}
\end{aligned}
$$

If the incident electromagnetic wave is polarised in the perpendicular direction, $\mathbf{A}=\left(0, A_{y}, 0\right)$, the expressions for the electromagnetic fields are:

$$
\begin{aligned}
E_{x}^{\mathrm{s}} & =\frac{1}{i \omega \varepsilon} \frac{\partial^{2} A_{y}}{\partial x \partial y} \quad H_{x}^{\mathrm{s}}=-\frac{\partial A_{y}}{\partial z} \\
E_{y}^{\mathrm{s}} & =-i \omega \mu A_{y}+\frac{1}{i \omega \varepsilon} \frac{\partial^{2} A_{y}}{\partial y^{2}} \quad H_{y}^{\mathrm{s}}=0 \\
E_{z}^{\mathrm{s}} & =\frac{1}{i \omega \varepsilon} \frac{\partial^{2} A_{y}}{\partial z \partial y} \quad H_{x}^{\mathrm{s}}=-\frac{\partial A_{y}}{\partial x}
\end{aligned}
$$

Using the principle of superposition we can add the effects of both polarizations and calculate the fields induced by the presence of the 3-D body.

$$
\begin{aligned}
E_{x}^{\mathrm{s}} & =-i \omega \mu A_{x}+\frac{1}{i \omega \varepsilon} \frac{\partial^{2} A_{x}}{\partial x^{2}}+\frac{1}{i \omega \varepsilon} \frac{\partial^{2} A_{y}}{\partial x \partial y} \quad H_{x}^{\mathrm{s}}=-\frac{\partial A_{y}}{\partial z} \\
E_{y}^{\mathrm{s}} & =-i \omega \mu A_{y}+\frac{1}{i \omega \varepsilon} \frac{\partial^{2} A_{y}}{\partial y^{2}}+\frac{1}{i \omega \varepsilon} \frac{\partial^{2} A_{x}}{\partial y \partial x} \quad H_{y}^{\mathrm{s}}=-\frac{\partial A_{x}}{\partial z} \\
E_{z}^{\mathrm{s}} & =\frac{1}{i \omega \varepsilon} \frac{\partial^{2} A_{x}}{\partial z \partial x}+\frac{1}{i \omega \varepsilon} \frac{\partial^{2} A_{y}}{\partial z \partial y} \quad H_{z}^{\mathrm{s}}=-\frac{\partial A_{y}}{\partial x}-\frac{\partial A_{x}}{\partial y}
\end{aligned}
$$

The solution of this set of equations must be obtained numerically (Habashy et al., 1993). 
In order to gain insight into the physical meaning of the set of equations 3,4 and 5, we consider a simple but illustrative example. We shall consider a 3-D body embedded in a uniform full space in which the induced currents inside it can be approximated by a current of intensity $I$ over an incremental length $l$. Taking this current to be $x$-directed, the vector potential will only have a nonzero $x$-component. It can be demonstrated (Harrington, 1961) that the $x$-component of the vector potential is:

$$
A_{x}=\frac{I l}{4 \pi|\mathbf{r}|} \mathrm{e}^{-i k|\mathbf{r}|}
$$

where $|\mathbf{r}|$ is the distance between the 3-D structure and the point at the surface where we are making measurements. Substituting this value into Equation 5 and retaining only the main terms, we may write:

$$
\begin{aligned}
E_{x}^{\mathrm{s}}= & -i \omega \mu \frac{I l}{4 \pi|\mathbf{r}|} \mathrm{e}^{-i k|\mathbf{r}|}-\frac{1}{i \omega \varepsilon} \frac{I l}{4 \pi} \frac{k^{2} x^{2}}{|\mathbf{r}|^{3}} \mathrm{e}^{-i k|\mathbf{r}|}+\mathrm{o}\left(<|\mathbf{r}|^{-4}\right) \\
& \approx-i \omega \mu \frac{I l}{4 \pi|\mathbf{r}|} \mathrm{e}^{-i k|\mathbf{r}|}-\frac{\mu \sigma}{\varepsilon} \frac{I l}{4 \pi} \frac{x^{2}}{|\mathbf{r}|^{3}} \mathrm{e}^{-i k|\mathbf{r}|} \\
E_{y}^{\mathrm{s}} & =-\frac{1}{i \omega \varepsilon} \frac{I l}{4 \pi} \frac{k^{2} x y}{|\mathbf{r}|^{3}} \mathrm{e}^{-i k|\mathbf{r}|}+\mathrm{o}\left(<|\mathbf{r}|^{-4}\right) \approx-\frac{\mu \sigma}{\varepsilon} \frac{I l}{4 \pi} \frac{x y}{|\mathbf{r}|^{3}} \mathrm{e}^{-i k|\mathbf{r}|} \\
E_{z}^{\mathrm{s}} & =-\frac{1}{i \omega \varepsilon} \frac{I l}{4 \pi} \frac{k^{2} x z}{|\mathbf{r}|^{3}} e^{-i k|\mathbf{r}|}+\mathrm{o}\left(<|\mathbf{r}|^{-4}\right) \approx-\frac{\mu \sigma}{\varepsilon} \frac{I l}{4 \pi} \frac{x z}{|\mathbf{r}|^{3}} \mathrm{e}^{-i k|\mathbf{r}|} \\
H_{x}^{\mathrm{s}} & =0 \quad H_{y}^{\mathrm{s}} \approx \frac{i k z}{|\mathbf{r}|^{2}} \frac{I l}{4 \pi} \mathrm{e}^{-i k|\mathbf{r}|} \quad H_{z}^{\mathrm{s}} \approx \frac{i k y}{|\mathbf{r}|^{2}} \frac{I l}{4 \pi} \mathrm{e}^{-i k|\mathbf{r}|}
\end{aligned}
$$

Simplifying the last equation leads to:

$$
\begin{aligned}
E_{x}^{\mathrm{s}} & =-i \omega \mu A_{x}-\frac{\mu \sigma}{\varepsilon} \frac{x^{2}}{|\mathbf{r}|^{2}} A_{x} \quad E_{y}^{\mathrm{s}}-\frac{\mu \sigma}{\varepsilon} \frac{x y}{|\mathbf{r}|^{2}} A_{x} \quad E_{z}^{\mathrm{s}}=-\frac{\mu \sigma}{\varepsilon} \frac{x z}{|\mathbf{r}|^{2}} A_{x}^{\mathrm{s}} x \\
& \left.=0 \quad H_{y}^{\mathrm{s}}=\frac{i k z}{|\mathbf{r}|} A_{x} \quad H_{z}^{\mathrm{s}}=\frac{i k y}{|\mathbf{r}|} A_{x}\right)
\end{aligned}
$$

In the electric field expressions, the term $\left(-i \omega \mu A_{x}\right)$ is called the inductive part and the term $\left(-\frac{\mu \sigma}{\varepsilon} \frac{x^{2}}{\mid \mathbf{r}^{2}} A_{x}\right)$ is the galvanic one (Jackson, 1975; Habashy et al., 1993). The induction term depends on the volume currents inside the 3-D body (and hence on the size of the body and the conductivity contrast between the 3-D body and the regional structure in which it is embedded). Its amplitude depends linearly on the frequency; for low frequencies this term will tend to zero. On the other hand, the amplitude of the galvanic term is frequency independent. The magnetic field amplitude depends on the size of the body and the conductivity contrast, and varies linearly with $\sqrt{\omega}$. 
To gain further insight and to analyze the importance of the inductive and galvanic terms in Equation 5, we have calculated the numerical response of a 3 -D body embedded in a homogenous medium of $500 \Omega \mathrm{m}$ using the 3-D code of Mackie et al. (1994). The response of the model was calculated for two cases: first, when the resistivity of the 3-D body is $1 \Omega \mathrm{m}$, and second for a resistivity of $250,000 \Omega \mathrm{m}$. The fields along a profile crossing the 3-D bodies transversely ( $x$-direction) are presented in Figure 2 for a period $(T)$ of $10 \mathrm{~s}$. Since the 3-D body is elongated in the $y$-direction, the inductive term

Hx-field

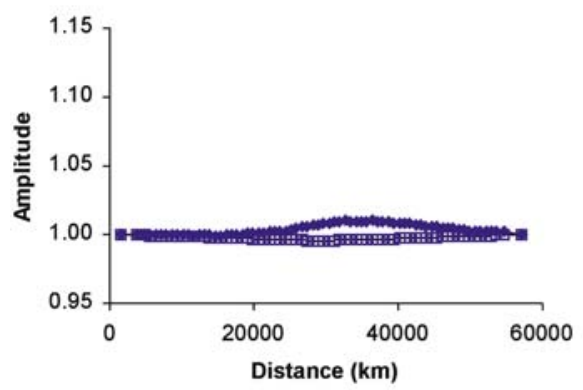

Hy-field

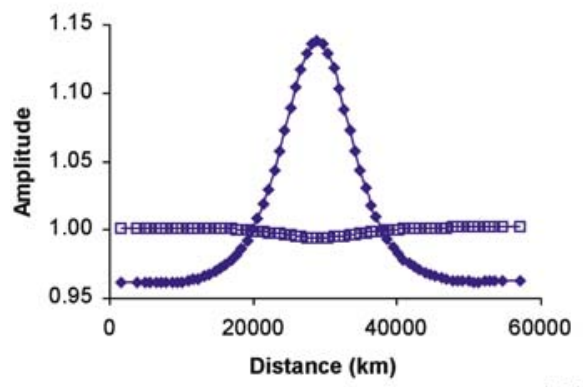

Hz-field $\quad \rightarrow-1$ Ohm.m
Ex-field

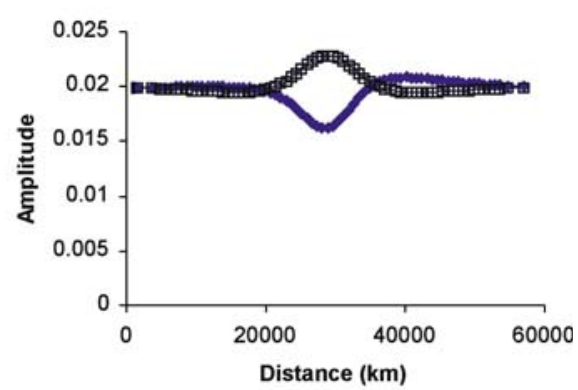

Ey-field

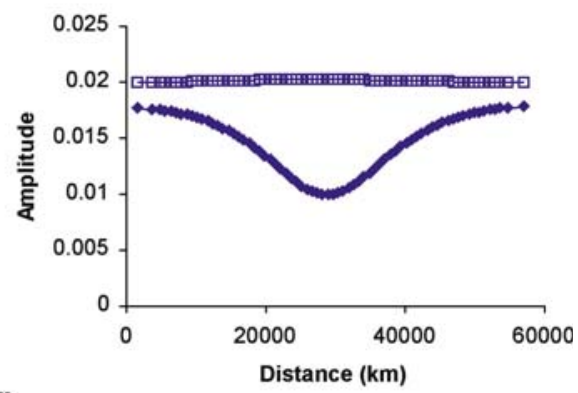

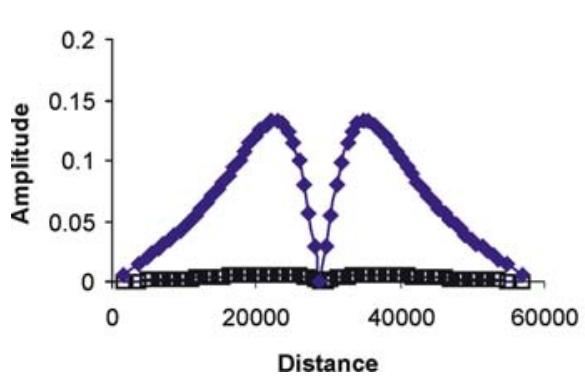

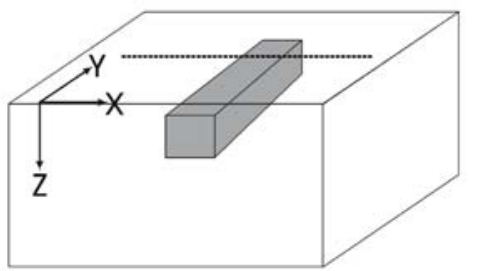

Figure 2. Comparison of inductive and galvanic effects depending on the resistivity contrast between the $3-\mathrm{D}$ body and the regional medium at $T=10 \mathrm{~s}$. 
$-i \omega \mu A_{y} \gg i \omega \mu A_{x}$, and since the profile is far away from the lateral boundaries of the body ( $y$-direction), the galvanic term along the $x$-direction is more important than that in the $y$-direction.

It can be observed in Figure 2 that the $x$-direction electric fields in both cases are symmetric (with respect to the $x$-axis), and that the galvanic term is more important than the inductive one. For both models, the $|\Delta \log \rho|$ between the 3-D body and the surrounding space is the same, producing the symmetry observed in the $E_{x}$ fields; where $\rho$ is the resistivity. On the other hand, the $y$-direction electric fields for both models display a strong difference since the galvanic effects are weaker than the inductive ones (current volumes circulating inside the 3-D body). In the less resistive 3-D body, the volume currents are much more important than in the more resistive case. The $x$-direction magnetic fields are practically symmetric in both cases and less affected than the magnetic field in the $y$-direction. The $y$ - and the verticalcomponents of the magnetic field for the less resistive 3-D body are much more affected than in the case of a 3-D resistive body.

Both inductive and galvanic effects will change the regional response in the presence of conductive 3-D structures; if the 3-D body is more resistive than the surrounding medium, the regional response will be affected mainly by galvanic distortion.

\section{Geophysical Aspects of 2-D Interpretation of Quasi 2-D Data}

We define a quasi 2-D structure as a 2-D regional-scale structure with the presence of medium-scale 3-D bodies. The main goal of MT interpretation of quasi-2-D data with 2-D techniques is to recover the maximum number of features of the regional 2-D structure without introducing artifacts due to 3-D effects. Although the response of the 3-D medium-scale bodies cannot be removed by the use of tensor decomposition techniques, the data still must be corrected for near-surface galvanic distortions. The importance of removing galvanic distortion effects on 3-D data before its 2-D or 3-D interpretation has been shown by Ledo et al. (1998) and Ledo et al. (2002a). A review of the proposed methods to correct galvanic distortions on 3-D data can be found in Ogawa (2002). Here we restrict our attention to data not affected by nearsurface galvanic distortion.

At each site and for all of the period range over which data are acquired, the feasible data to use during a 2-D interpretation are separated into two modes: transverse magnetic (TM) mode (currents crossing strike) and transverse electric (TE) mode (currents flowing parallel to strike). In both modes, we have apparent resistivities and phases relating the horizontal EM fields, and for the TE mode there is also the magnetic induction vector data. Our objective in this section is to determine the limits on the modes, period and spatial ranges that can be used during the 2-D interpretation of 3-D data. Perhaps the most 
accepted paradigm in the interpretation of 3-D data is emphasis on the TM mode in the presence of 3-D conductive structures and the TE-mode when the 3-D structure is resistive (Ting and Hohmann, 1981; Wannamaker et al., 1984a, b, 1991; Berdichevsky, 1999). This approach allows the determination of reasonable geological models in many cases, although it leads to incorrect interpretations in some situations. We will describe different cases in order to constrain the limitations of this assumption.

All the examples in this section present the same line of reasoning. First we derive the general expression for the TM and TE impedance tensor modes from Equation 5, then we compare these results with a synthetic model, and we finish with examples from real data when available. We have used the same 2-D synthetic model for all of the examples presented here (Figure 3). The numerical responses of all of the 3-D models presented in this review have been calculated using the 3D code of Mackie et al. (1994) with modifications by Mackie and Booker (2000, personal communication.)

\subsection{CASE A}

First, we consider a 2-D regional model with a 3-D body embedded in it perpendicular to the 2-D structures (Figure 4). Given the location of the measurement point (far away from the extremes of the body) we can assume that the variation of the vector potential due to the 3-D structure along the $y$-direction is negligible, $\partial_{y} \mathbf{A} \cong 0$.

Considering now the anomalous fields generated by two perpendicularly polarized electromagnetic fields, and using the superposition principle, the anomalous fields can be written from Equation 5 as follows:

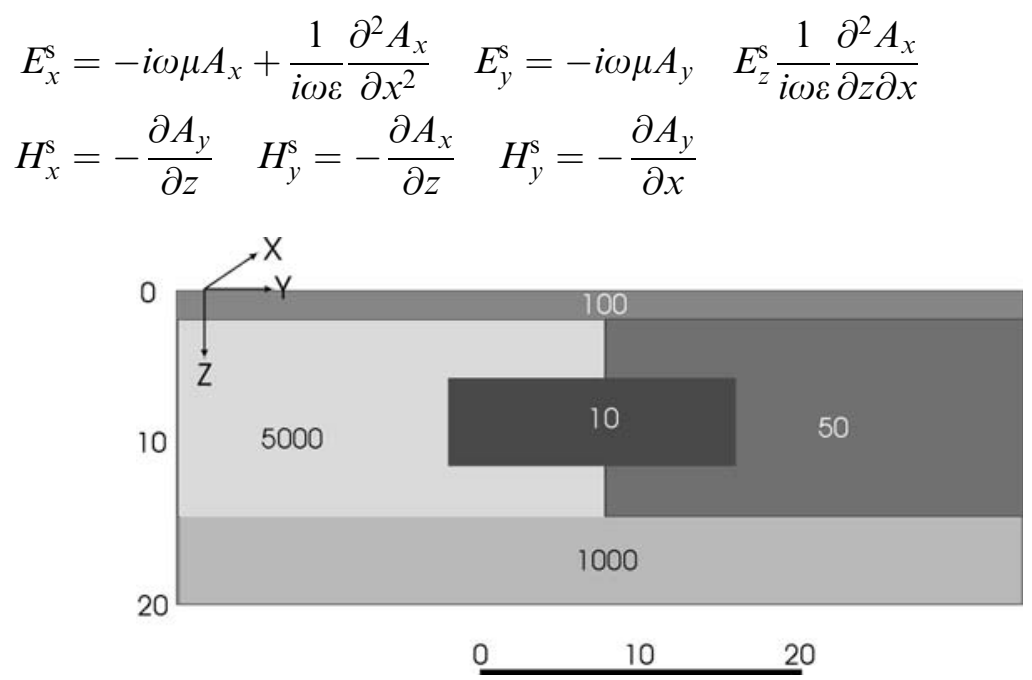

Figure 3. 2-D regional model. Distances are shown in $\mathrm{km}$ and resistivities in $\Omega \mathrm{m}$. 


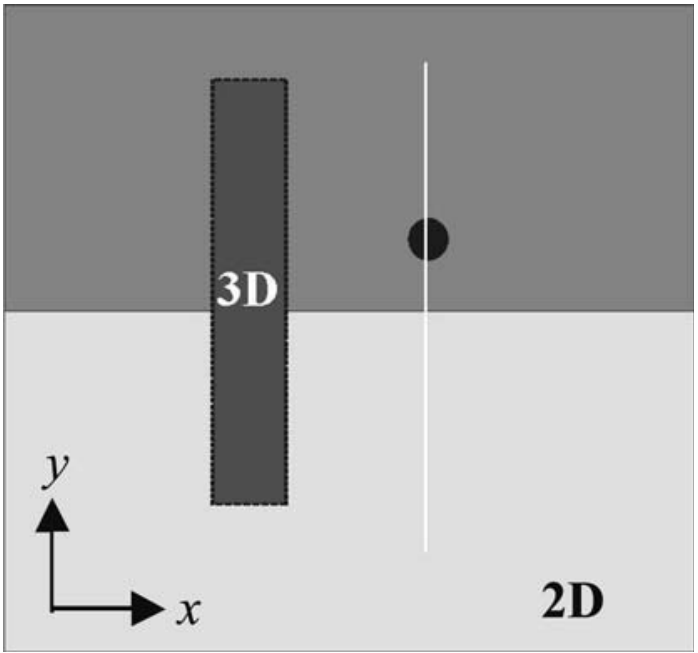

Figure 4. Plan view of a generic 2-D model with a 3-D structure. Black circle: measurement site. White line: data profile.

Since the $y$-dimension of the 3-D body is much bigger than the $x$ dimension and the induction term depends on the dimensions of the 3-D body, we can consider that $A_{y} \gg A_{x}$. Thus, the electric field in the $x$-direction is affected mainly by galvanic effects due to the accumulation of charge on surfaces of the 3-D body parallel to the $y$-direction. On the other hand, the electric field in the $y$-direction is affected by an inductive term due to the $y$ directed currents within the 3-D body. For the magnetic fields, a similar argument leads us to consider that $H_{x}^{\mathrm{s}} \gg H_{y}^{\mathrm{s}}$.

The measured field can be expressed as the sum of the 2-D regional ones plus the anomalous fields as:

$$
\begin{array}{ll}
E_{x}^{\mathrm{t}}=E_{x}^{2 \mathrm{D}}+\left(E_{x}^{\mathrm{s}}\right)_{\text {galvanic }}=g E_{x}^{2 \mathrm{D}} & H_{x}^{\mathrm{t}}=H_{x}^{2 \mathrm{D}}+H_{x}^{\mathrm{s}} \\
E_{y}^{\mathrm{t}}=E_{y}^{2 \mathrm{D}}+\left(E_{y}^{\mathrm{s}}\right)_{\text {inductive }} & H_{y}^{\mathrm{t}}=H_{y}^{2 \mathrm{D}} \\
& H_{z}^{\mathrm{t}}=H_{z}^{2 \mathrm{D}}+H_{z}^{\mathrm{s}}
\end{array}
$$

where $g$ is a real, frequency independent scalar. The off-diagonal terms of the impedance tensor are:

$$
\begin{aligned}
& Z_{x y}^{\mathrm{t}}=Z_{\mathrm{TE}}^{\mathrm{t}}=\frac{g E_{x}^{2 \mathrm{D}}}{H_{y}^{2 \mathrm{D}}}=g Z_{\mathrm{TE}}^{2 \mathrm{D}} \\
& Z_{y x}^{\mathrm{t}}=Z_{\mathrm{TM}}^{\mathrm{t}}=\frac{E_{y}^{2 \mathrm{D}}+\left(E_{y}^{\mathrm{s}}\right)_{\text {inductive }}}{H_{x}^{2 \mathrm{D}}+H_{x}^{\mathrm{s}}}
\end{aligned}
$$


If the 3-D body resistivity is higher than that of the surrounding 2-D regional structure, the anomalous inductive term of the electric field will be insignificant and the TM mode will present a weak inductive distortion, while the TE mode will be affected by galvanic distortion. On the other hand, if the 3-D body is less resistive than the embedding medium, the TM mode can be affected strongly by inductive effects. Thus, the TM mode is not appropriate for a 2-D interpretation in this case.
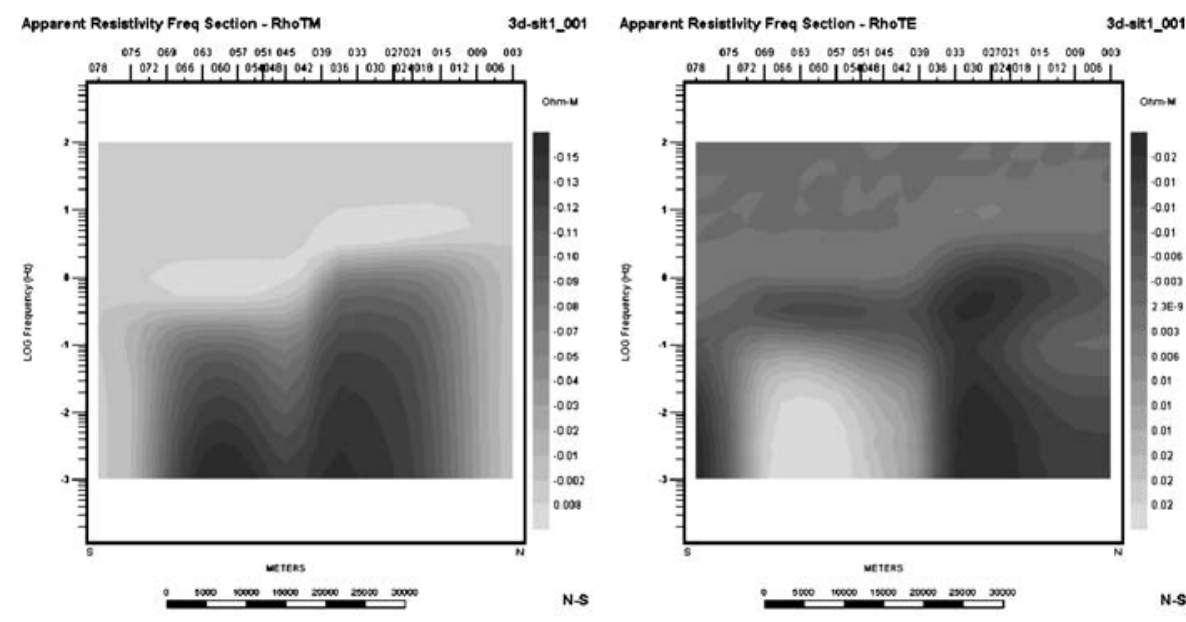

Phase Freq Section - PhstM

3d-sit1_001
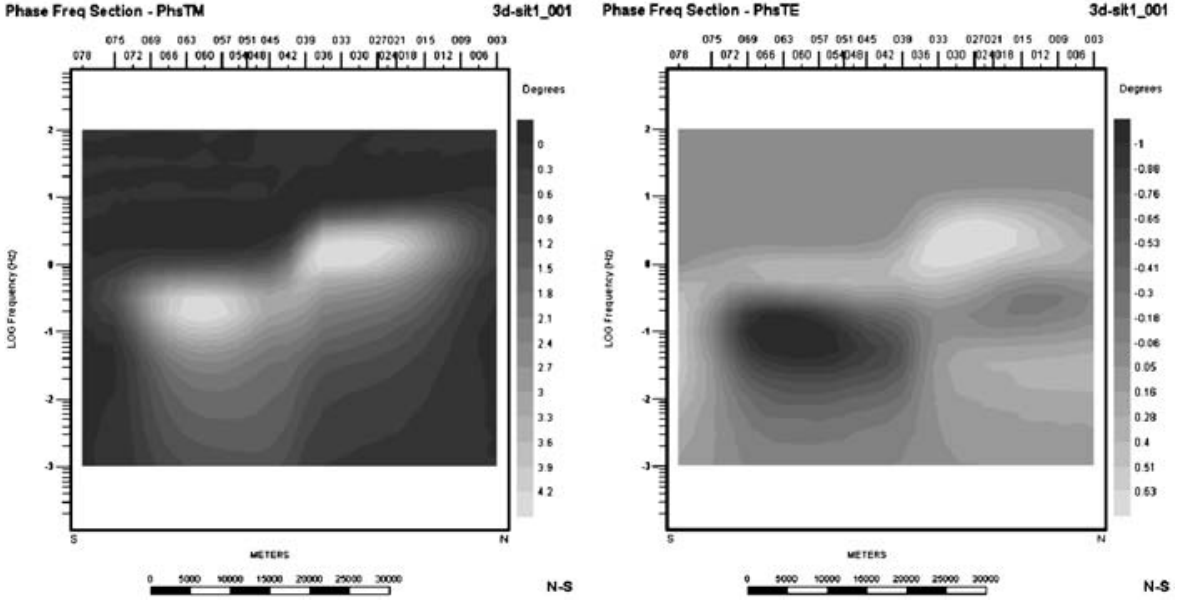

Figure 5. (a) Difference between the apparent resistivity (in $\log _{10}$ ) and phase data of the 2-D regional model and the quasi 2-D model for case A. Top right: apparent resistivity TE mode; Top left: apparent resistivity TM mode; Bottom right: phase TE mode; Bottom left: phase TM mode. (b) Difference between the tipper components for the 2-D regional model and the quasi 2-D model for case A. Top right: real part of transfer function in $y$-direction; Top left: imaginary part of transfer function in $y$-direction; Bottom right: real part of transfer function in $x$-direction; Bottom left: imaginary part of transfer function in $x$-direction. 
To illustrate numerically these results we have embedded a 3-D conductive structure in our 2-D regional model. The structure is elongated along the $y$-axis and intersects the 2-D structure at a right angle. Figure $5 \mathrm{a}$ and $\mathrm{b}$ show the apparent resistivity, phase and tipper pseudosection differences between the 2-D regional responses and the 3-D responses for both modes along a profile in the $y$-direction. It is obvious that the TM mode is more affected. The differences observed correspond to a 7\% change in the impedance tensor components. The tipper components are practically unaffected in this case (Figure $5 b$ ).

We note also that the effect of the 3-D body in the TM mode starts at very short periods, and the phases denote its presence at shorter periods than does the apparent resistivity.

The complete magnetotelluric study of the Nanga Parbat in northern Pakistan by Park and Mackie $(1997,2000)$ is a good example of this case
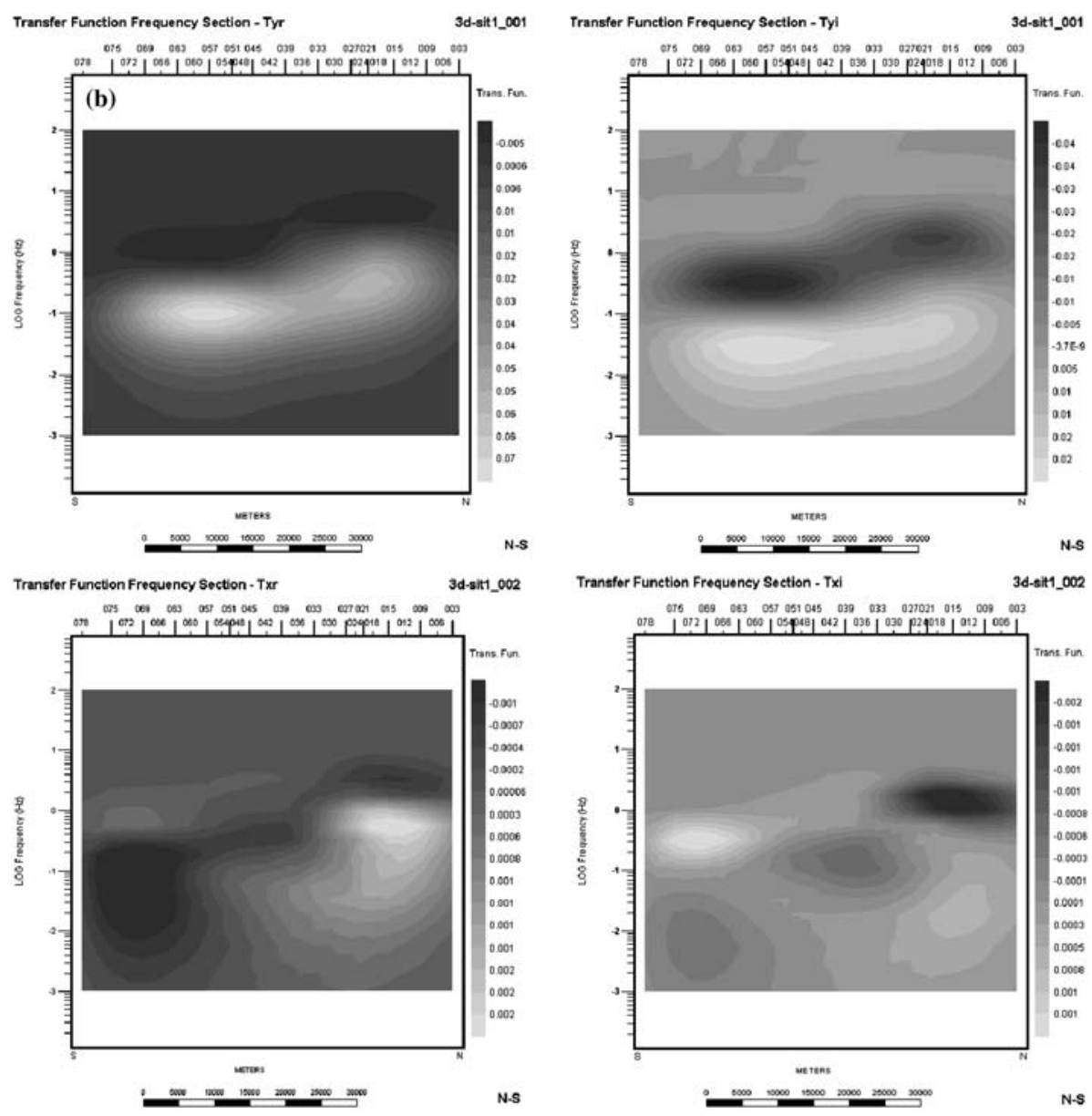

Figure 5. (continued) 

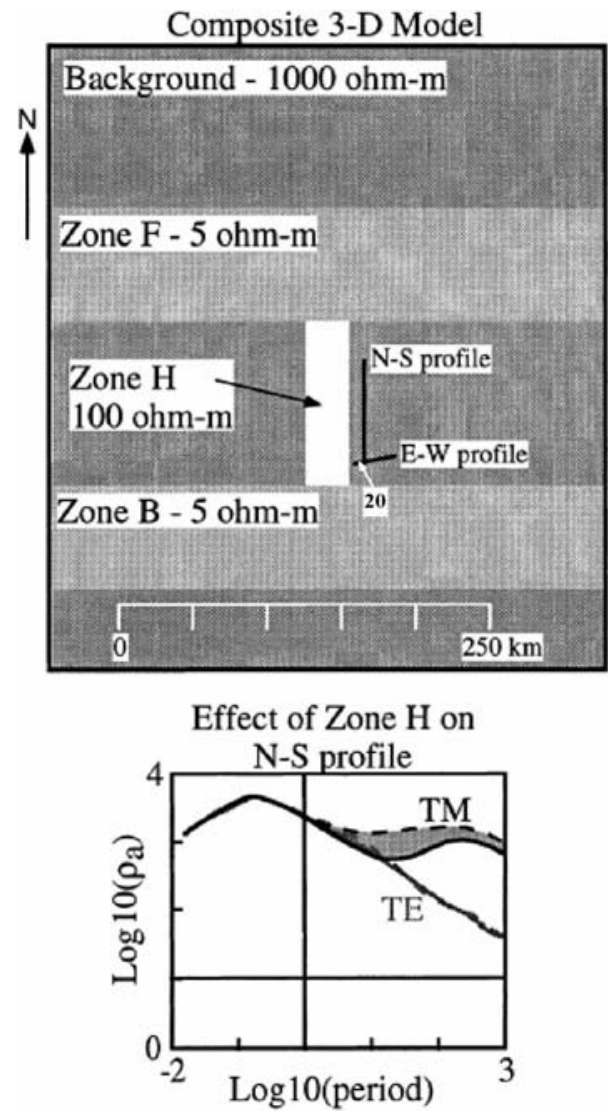

Figure 6. Composite 3-D model (slice at $12 \mathrm{~km}$ is pictured). Responses at site 20 are shown as solid lines. The effect of removing zone $\mathrm{H}$ from the pictured model is shown by dashed lines and consists of raising the TM $(\mathrm{N}-\mathrm{S})$ mode by a maximum factor of 2.5 at $30 \mathrm{~s}$. The TE $(\mathrm{E}-\mathrm{W})$ mode is unaffected. The period is given is seconds and $\rho_{\mathrm{a}}$ is the apparent resistivity in $\Omega \mathrm{m}$ (From Park and Mackie, 2000).

(Figure 6). Their study also presented a methodology to evaluate the validity of the 2-D assumption by using 3-D modeling. The comparison between 2-D and 3-D modeling reveals that the main effect of a conducting structure (Zone $\mathrm{H}$ in their study, $100 \Omega \mathrm{m}$ ) parallel to a $\mathrm{N}-\mathrm{S}$ profile is to lower the apparent resistivity TM mode by a maximum factor of 2.5 and the phase by $5^{\circ}$ at $30 \mathrm{~s}$.

\subsection{CASE B}

Let us consider the determination of the impedance tensor on a site near a 3-D body edge (Figure 7). Using the superposition principle and Equation 5, the anomalous fields generated by two perpendicularly polarized electromagnetic fields may be written as: 


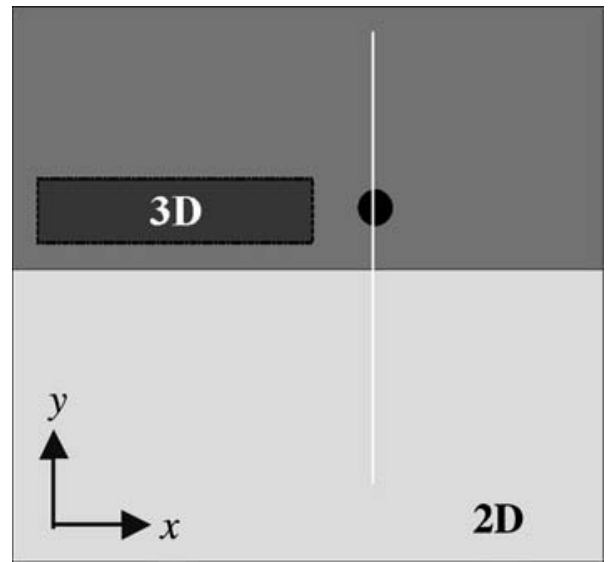

Figure 7. Plan view of a generic 2-D model with a 3-D structure. Black circle: measurement site. White line: data profile.

$$
\begin{aligned}
& E_{x}^{\mathrm{s}}=-i \omega \mu A_{x}+\frac{1}{i \omega \varepsilon} \frac{\partial^{2} A_{x}}{\partial x^{2}}+\frac{1}{i \omega \varepsilon} \frac{\partial^{2} A_{y}}{\partial x \partial y} \quad H_{x}^{\mathrm{s}}=-\frac{\partial A_{y}}{\partial z} \\
& E_{y}^{\mathrm{s}}=-i \omega \mu A_{y}+\frac{1}{i \omega \varepsilon} \frac{\partial^{2} A_{y}}{\partial y^{2}}+\frac{1}{i \omega \varepsilon} \frac{\partial^{2} A_{x}}{\partial y \partial x} \quad H_{y}^{\mathrm{s}}=-\frac{\partial A_{x}}{\partial z} \\
& E_{z}^{\mathrm{s}}=\frac{1}{i \omega \varepsilon} \frac{\partial^{2} A_{x}}{\partial z \partial x}+\frac{1}{i \omega \varepsilon} \frac{\partial^{2} A_{y}}{\partial z \partial y} \quad H_{z}^{\mathrm{s}}=-\frac{\partial A_{y}}{\partial x}-\frac{\partial A_{x}}{\partial y}
\end{aligned}
$$

Since the $x$-dimension of the body is much larger than the $y$-dimension and the induction term depends on the dimension of the 3-D body, we can consider that $A_{x} \gg A_{y}$. Thus, inductive and galvanic effects influence the electric field in the $x$-direction while the electric field in the $y$-direction is affected mainly by galvanic effects due to the accumulation of charge on the surfaces of the 3-D body. Simultaneously, the secondary magnetic field in the $y$-direction will be bigger than that in the $x$-direction. The total measured field may be expressed as:

$$
\begin{aligned}
E_{x}^{\mathrm{t}} & =E_{x}^{2 \mathrm{D}}+\left(E_{x}^{\mathrm{s}}\right)_{\text {galvanic }}+\left(E_{x}^{\mathrm{s}}\right)_{\text {inductive }}=g E_{x}^{2 \mathrm{D}}+\left(E_{x}^{\mathrm{s}}\right)_{\text {inductive }} \\
E_{y}^{\mathrm{t}} & =E_{y}^{2 \mathrm{D}}+\left(E_{y}^{\mathrm{s}}\right)_{\text {galvanic }}=g E_{y}^{2 \mathrm{D}} \\
H_{x}^{\mathrm{t}} & =H_{x}^{2 \mathrm{D}} \quad H_{y}^{\mathrm{t}}=H_{y}^{2 \mathrm{D}}+H_{y}^{\mathrm{s}} \quad H_{z}^{\mathrm{t}}=H_{z}^{2 \mathrm{D}}+H_{z}^{\mathrm{s}} \\
Z_{x y}^{\mathrm{t}} & =Z_{\mathrm{TE}}^{\mathrm{t}}=\frac{g E_{x}^{2 \mathrm{D}}+\left(E_{x}^{\mathrm{s}}\right)_{\text {inductive }}}{H_{y}^{2 \mathrm{D}}+H_{y}^{\mathrm{s}}} \\
Z_{y x}^{\mathrm{t}} & =Z_{\mathrm{TM}}^{\mathrm{t}}=\frac{g E_{y}^{2 \mathrm{D}}}{H_{x}^{2 \mathrm{D}}}=g Z_{\mathrm{TM}}^{\mathrm{t}}
\end{aligned}
$$



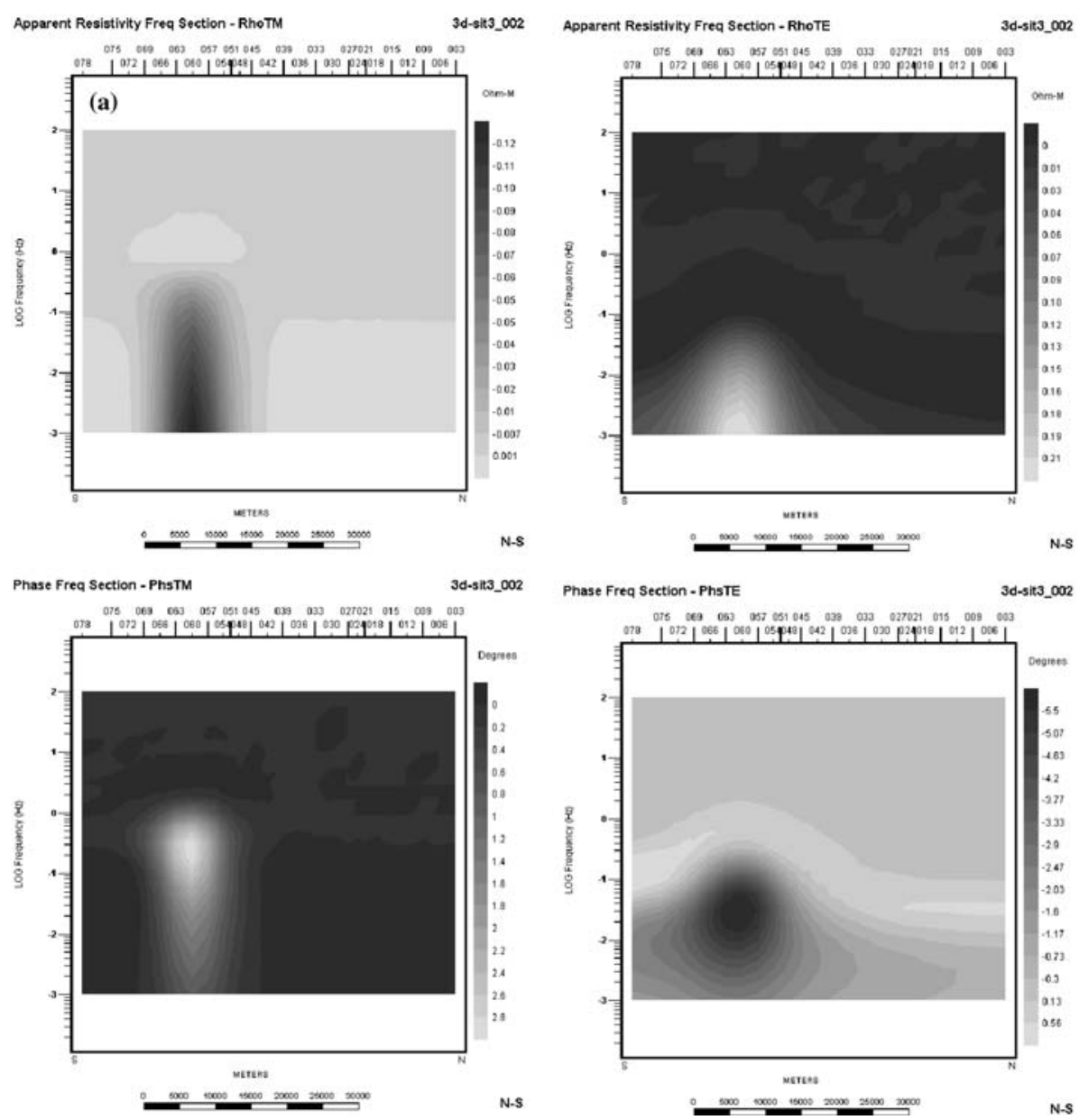

Figure 8. (a) Difference between the 2-D regional data and the quasi 2-D data for case B. Top right: apparent resistivity TE mode; Top left: apparent resistivity TM mode; Bottom right: phase TE mode; Bottom left: phase TM mode. (b) Difference between the 2-D regional data and the quasi 2-D data for case B. Top right: real part of transfer function in $y$-direction; Top left: imaginary part of transfer function in $y$-direction; Bottom right: real part of transfer function in $x$-direction; Bottom left: imaginary part of transfer function in $x$-direction.

In this case, if the measuring point is far away from the edges of the 3-D body (y-direction) the most disturbed mode will be the TE. If the 3-D body presents a resistivity higher than the surrounding media, the secondary electric inductive term will be weaker. Therefore, the TE mode will be influenced mainly by galvanic distortion. If the $y$-location of the measuring point is similar to the 3-D body $y$-location, the TM mode is the most affected.

Figure $8 \mathrm{a}$ and $\mathrm{b}$ display the apparent resistivity, phase and tipper pseudosection differences between the 2-D regional responses and the 3-D 


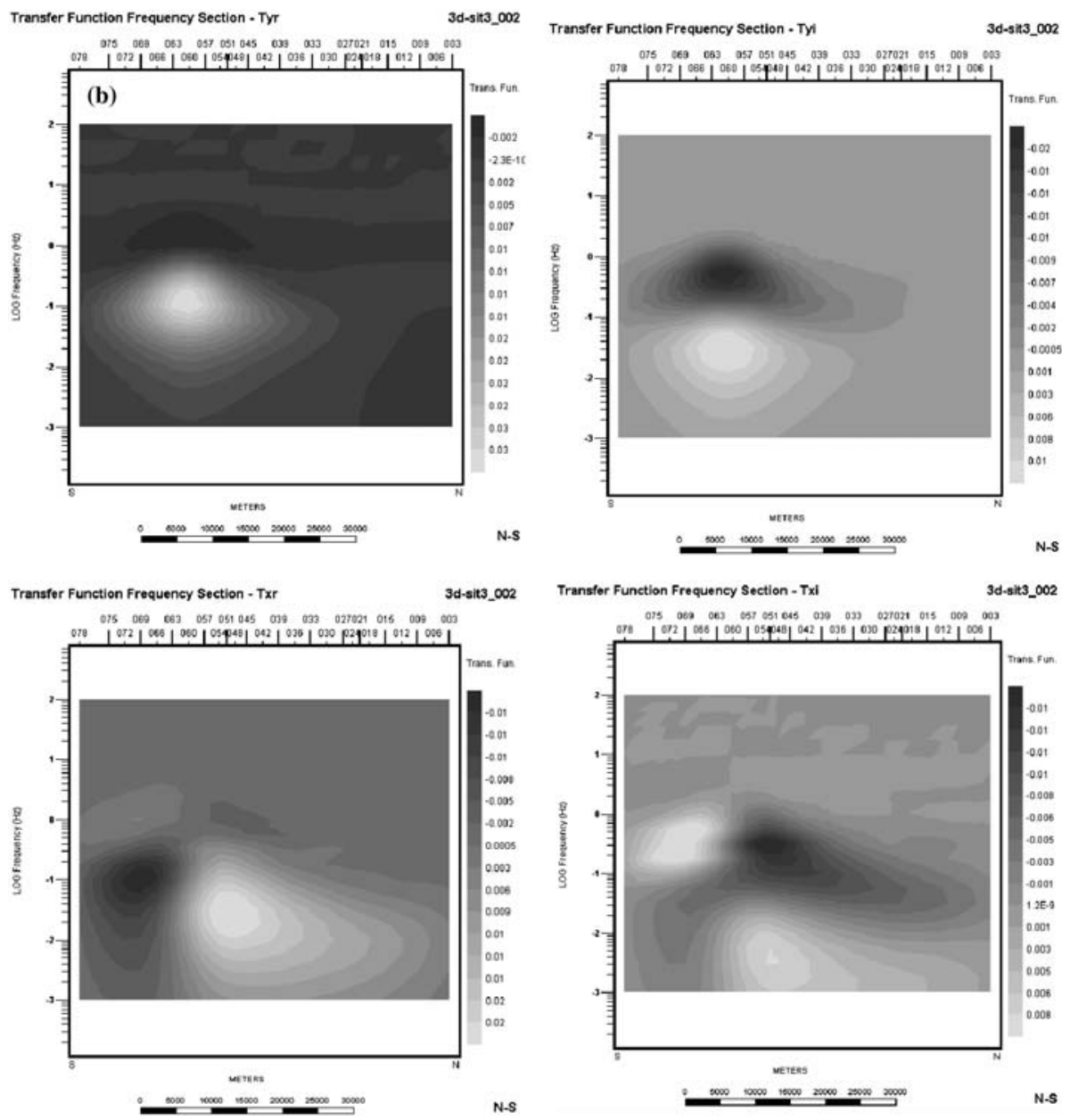

Figure 8. (continued)

responses for both modes along a profile in the $y$-direction. The TE mode is the most affected by the presence of the 3-D body, although at longer periods than the TM mode is. Moreover, the anomaly in the TE mode shows a longer wavelength with more sites being affected. The difference in the phase responses for the TE mode in both cases reach a value of $5.7^{\circ}$, which corresponds to a $10 \%$ error in the impedance tensor components.

\subsection{CASE C}

In this case, the 3-D anomalous body is parallel to the 2-D regional strike and the measured profile crosses it (Figure 9). We can consider that the variation 


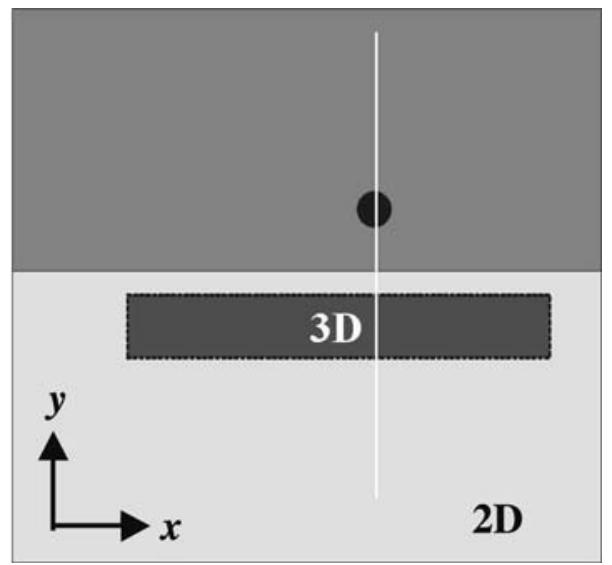

Figure 9. Plan view of a generic 2-D model with a 3-D structure. Black circle: measurement site. White line: data profile.

of the vector potential due to the 3 -D structure along the $x$-direction is negligible $\left(\partial_{x} \mathbf{A} \cong 0\right.$ at large distances from the lateral edges of the 3-D body). Therefore, Equation 5 is written as:

$$
\begin{aligned}
E_{x}^{\mathrm{s}} & =-i \omega \mu A_{x} \quad H_{x}^{\mathrm{s}}=-\frac{\partial A_{y}}{\partial z} \\
E_{y}^{\mathrm{s}} & =-i \omega \varepsilon A_{y}+\frac{1}{i \omega \varepsilon} \frac{\partial^{2} A_{y}}{\partial y^{2}} \quad H_{y}^{\mathrm{s}}=-\frac{\partial A_{x}}{\partial z} \\
E_{z}^{\mathrm{s}} & =\frac{1}{i \omega \varepsilon} \frac{\partial^{2} A_{y}}{\partial z \partial y} \quad H_{z}^{\mathrm{s}}=-\frac{\partial A_{x}}{\partial y}
\end{aligned}
$$

It follows from previous considerations that $\mathrm{A}_{x} \gg A_{y}$, and thus the total fields can be written as:

$$
\begin{aligned}
E_{x}^{\mathrm{t}} & =E_{x}^{2 \mathrm{D}}+\left(E_{x}^{\mathrm{s}}\right)_{\text {inductive }}=E_{x}^{2 \mathrm{D}}+\left(E_{x}^{\mathrm{s}}\right)_{\text {inductive }} \\
E_{y}^{\mathrm{t}} & =E_{y}^{2 \mathrm{D}}+\left(E_{y}\right)_{\text {galvanic }}=g E_{y}^{2 \mathrm{D}} \\
H_{x}^{\mathrm{t}} & =H_{x}^{2 \mathrm{D}} \quad H_{y}^{\mathrm{t}}=H_{y}^{2 \mathrm{D}}+H_{y}^{\mathrm{s}} \quad H_{z}^{\mathrm{t}}=H_{z}^{2 \mathrm{D}}+H_{z}^{\mathrm{s}} \\
Z_{x y}^{\mathrm{t}} & =Z_{\mathrm{TE}}^{\mathrm{t}}=\frac{E_{x}^{2 \mathrm{D}}+\left(E_{x}^{2 \mathrm{D}}\right)}{H_{y}^{2 \mathrm{D}}+H_{y}^{\mathrm{s}}} \\
Z_{y x}^{\mathrm{t}} & =Z_{\mathrm{TE}}^{\mathrm{t}}=\frac{g E_{x}^{2 \mathrm{D}}}{H_{x}^{2 \mathrm{D}}}=g Z_{\mathrm{TM}}^{\mathrm{t}}
\end{aligned}
$$

In this case the TM mode is more robust to the presence of 3-D structure. Figure 10a and $\mathrm{b}$ present the apparent resistivity, phase and tipper pseudosection differences between the 2-D regional responses and the 3-D responses for both modes. The TM mode is less affected and also the number 

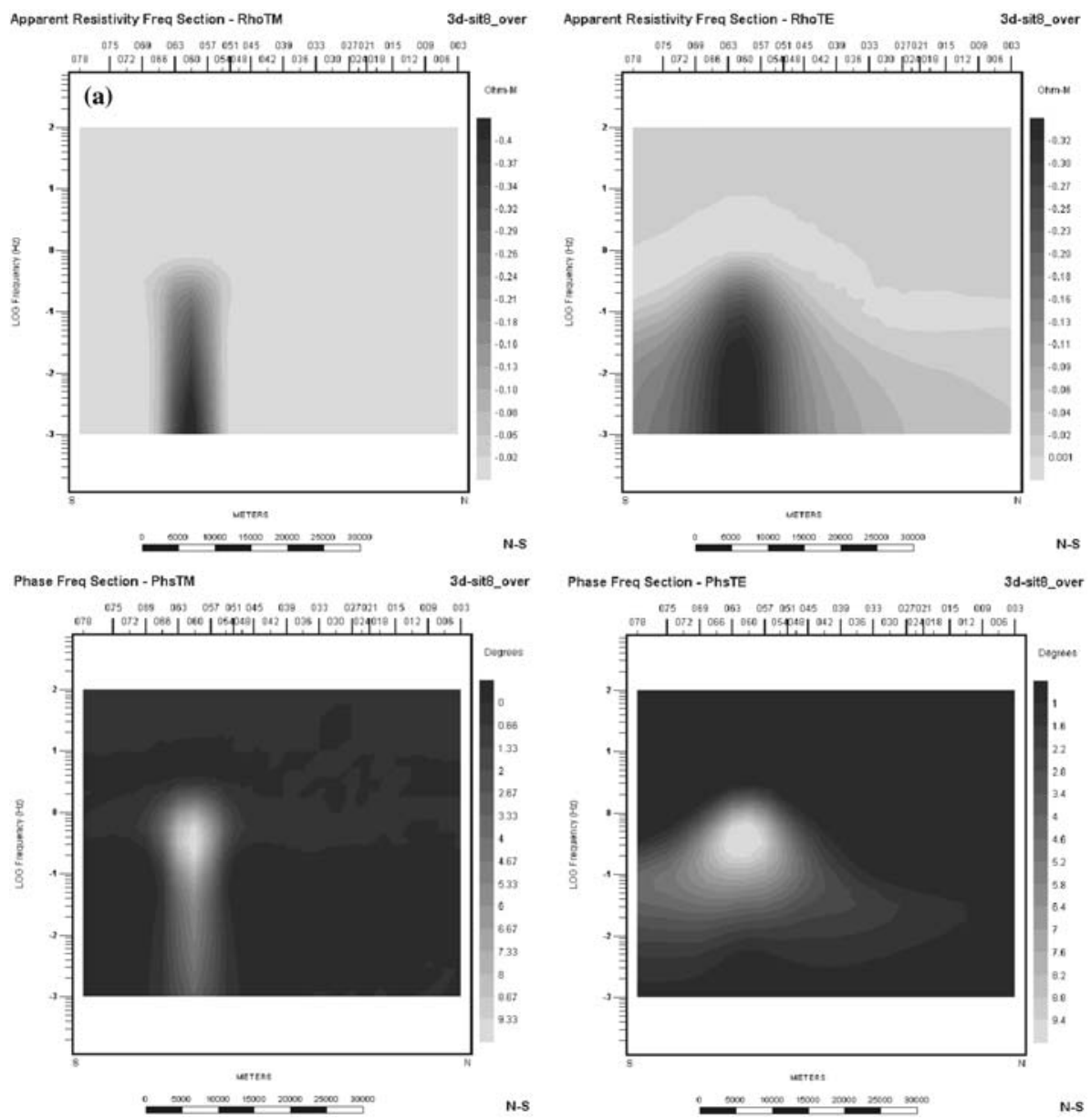

Figure 10. (a) Difference between the apparent resistivities and phases of the 2-D regional model and the quasi 2-D model for case C. Top left: apparent resistivity TM mode; Bottom right: phase TE mode; Bottom left: phase TM mode. (b) Difference between the tipper components of the 2-D regional model and the quasi 2-D model for case C. Top right: real part of transfer function in $y$-direction; Top left: imaginary part of transfer function in $y$-direction; Bottom right: real part of transfer function in $x$-direction; Bottom left: imaginary part of transfer function in $x$-direction.

of sites distorted by the presence of the 3-D structure is smaller than for the TE mode.

The magnetotelluric study of the Long Valley caldera magmatic system by Wannamaker et al. (1991) along an E-W profile is a good example of an MT profile crossing a 3-D conductive structure. The observed TE apparent resistivities and the vertical magnetic field were greatly depressed in comparison with the calculated 2-D model responses. In this case, the abrupt termination of the conductive caldera sediments less than $10 \mathrm{~km}$ north and 

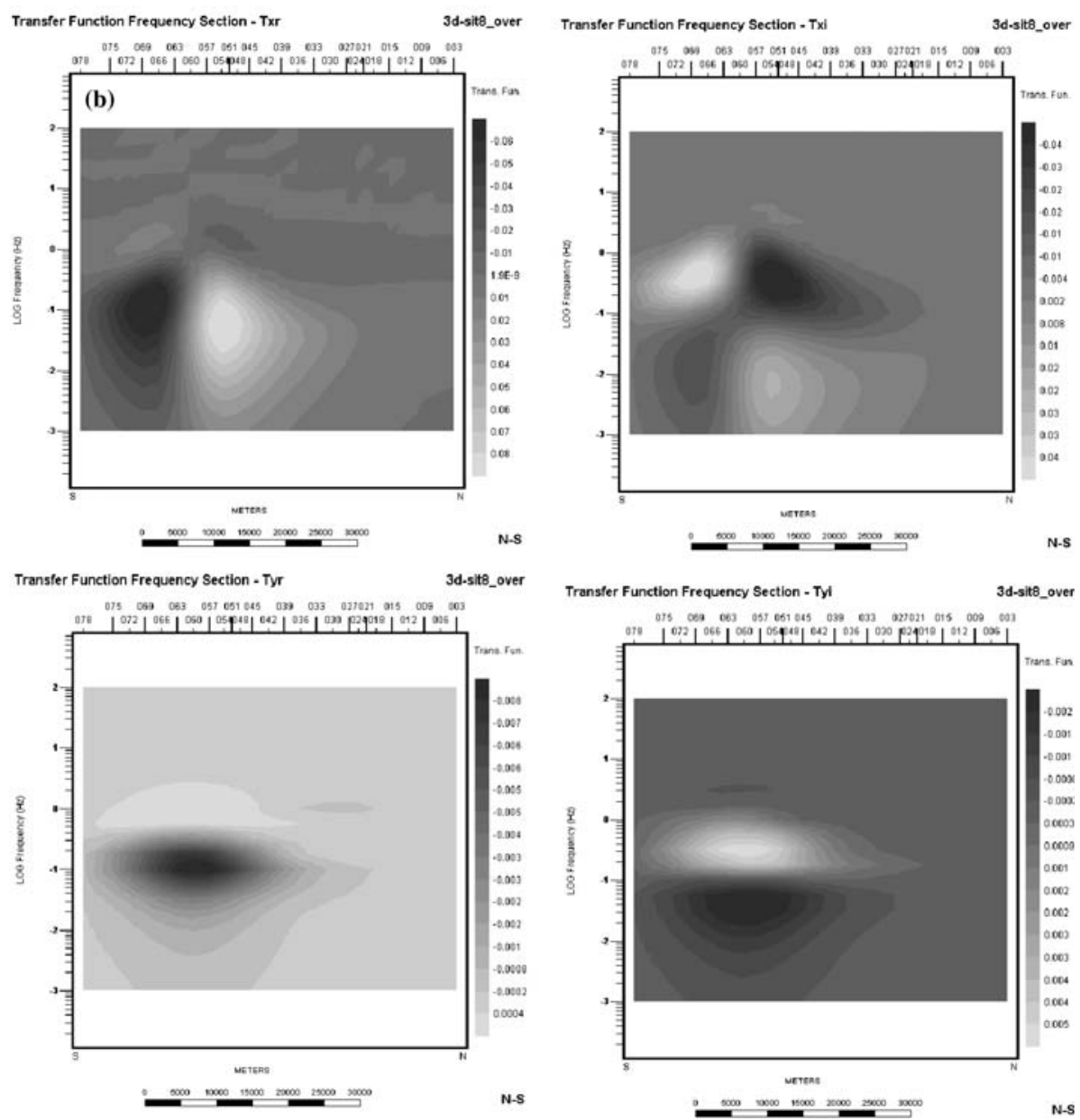

Figure 10. (continued)

south of the profile decreased the density of $\mathrm{N}-\mathrm{S}$ volume currents in the conductive sediments relative to a 2-D geometry. This hypothesis was tested and confirmed by Wannamaker (1999) through computing the numerical response of a 3-D model for the Long Valley caldera.

\subsection{CASE D}

For the last situation, we consider a 3-D structure cutting the $x$-axis of the $2-\mathrm{D}$ regional structure at an angle $\varphi$ (Figure 11). The reason for considering this model is to determine how the strike variation with depth affects the measured impedance tensor.

To calculate the anomalous fields generated by the 3-D structure we consider the $\left(x^{\prime}, y^{\prime}\right)$ coordinate system that forms an angle $\varphi$ with the $(x, y)$ 


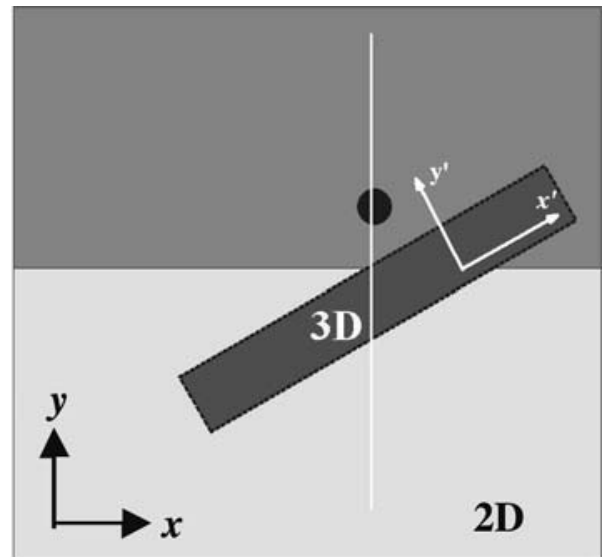

Figure 11. Plan view of a generic 2-D model with a 3-D structure tilted an angle of $45^{\circ}$. Black circle: measurement site. White line: data profile.

coordinate system. The fields generated by the 3-D body in the $\left(x^{\prime}, y^{\prime}\right)$ system can be rotated to the $(x, y)$ system. As discussed for Case $\mathrm{C}, \frac{\partial^{2} \mathbf{A}}{\partial x^{\prime 2}} \cong 0$ in the $\left(x^{\prime}, y^{\prime}\right)$ coordinate system. The volume currents in the $y^{\prime}$ direction can be considered minute in comparison with the volume currents in the $x^{\prime}$ direction (same hypothesis as in the previous case C).

$$
\begin{aligned}
& E_{x}^{\mathrm{s}}=\cos \varphi E_{x^{\prime}}^{\mathrm{s}}-\sin \varphi E_{y^{\prime}}^{\mathrm{s}} \approx-i \omega \mu A_{x^{\prime}} \cos \varphi-\frac{1}{i \omega \varepsilon} \frac{\partial^{2} A_{y^{\prime}}}{\partial y^{\prime 2}} \sin \varphi \\
& E_{y}^{\mathrm{s}}=\sin \varphi E_{x^{\prime}}^{\mathrm{s}}+\sin \varphi E_{y^{\prime}}^{\mathrm{s}} \approx-i \omega \mu A_{x^{\prime}} \sin \varphi-\frac{1}{i \omega \varepsilon} \frac{\partial^{2} A_{y^{\prime}}}{\partial y^{\prime 2}} \cos \varphi \\
& H_{x}^{\mathrm{s}}=\cos \varphi H_{x^{\prime}}^{\mathrm{s}}-\sin \varphi H_{y^{\prime}}^{\mathrm{s}} \approx \sin \varphi \frac{\partial A_{x^{\prime}}}{\partial x^{\prime}} \\
& H_{y}^{\mathrm{s}}=\sin \varphi H_{x^{\prime}}+\cos \varphi H_{y^{\prime}}^{\mathrm{s}} \approx-\cos \varphi \frac{\partial A_{x^{\prime}}}{\partial x^{\prime}} \\
& H_{z}^{\mathrm{s}}=-\frac{\partial A_{x}^{\prime}}{\partial x^{\prime}}-\frac{\partial A_{x}^{\prime}}{\partial y^{\prime}}
\end{aligned}
$$

The measured impedance can be expressed as:

$$
\begin{aligned}
E_{x}^{\mathrm{t}} & =E_{x}^{2 \mathrm{D}}+\cos \varphi\left(E_{x^{\prime}}^{\mathrm{s}}\right)_{\text {inductive }}-\sin \varphi\left(E_{y^{\prime}}^{\mathrm{s}}\right)_{\text {galvanic }}=g E_{x}^{2 \mathrm{D}}+\cos \varphi\left(E_{x^{\prime}}^{\mathrm{s}}\right)_{\text {inductive }} \\
E_{y}^{\mathrm{t}} & =E_{y}^{2 \mathrm{D}}+\sin \varphi\left(E_{x^{\prime}}^{\mathrm{s}}\right)_{\text {inductive }}+\cos \varphi\left(E_{y^{\prime}}^{\mathrm{s}}\right)=g E_{x}^{2 \mathrm{D}}+\sin \varphi\left(E_{x^{\prime}}^{\mathrm{s}}\right)_{\text {inductive }} \\
H_{x}^{\mathrm{t}} & =H_{x}^{2 \mathrm{D}}-\sin \varphi H_{y^{\prime}}^{\mathrm{s}} \\
H_{y}^{\mathrm{t}} & =H_{y}^{2 \mathrm{D}}+\cos \varphi H_{y^{\prime}}^{\mathrm{s}}
\end{aligned}
$$




$$
\begin{aligned}
& Z_{x y}^{\mathrm{t}}=Z_{\mathrm{TE}}^{\mathrm{t}}=\frac{g E_{x}^{2 \mathrm{D}}+\cos \varphi\left(E_{x^{\prime}}^{\mathrm{s}}\right)_{\text {inductive }}}{H_{x}^{2 \mathrm{D}}-\cos \varphi H_{y^{\prime}}^{\mathrm{s}}} \\
& Z_{y x}^{\mathrm{t}}=Z_{\mathrm{TM}}=\frac{g E_{y}^{2 \mathrm{D}}+\sin \varphi\left(E_{x^{\prime}}^{\mathrm{s}}\right)_{\text {inductive }}}{H_{x}^{2 \mathrm{D}}-\sin \varphi H_{y^{\prime}}^{\mathrm{s}}}
\end{aligned}
$$

As expected, the results obtained here with $\left(0<\varphi<90^{\circ}\right)$ are a combination of cases $\mathrm{A}$ and $\mathrm{C}$. In this instance, both polarizations are affected by the presence of the 3-D structure although, as the period becomes larger, the 3-D inductive effects vanish.

The conductive structure located in the center of the 2-D model (Figure 3) has been rotated $45^{\circ}$. The comparison between the $2-\mathrm{D}$ regional model responses and the quasi 2-D model is shown in Figure 12a and $b$.

There are several examples with real and synthetic data that can be associated with this situation, where the strike angle is depth dependent. It is worth noting that although a depth dependent strike could complicate or even invalidate a 2-D interpretation, useful geological information may still be obtained from analysis of the magnetotelluric data, as can be seen in the following examples.

The analysis of MT data in the Intermontane and Omineca Belts in the southern Canadian Cordillera by Marquis et al. (1995) showed a 0.01-0.1 s short period strike of predominantly $\mathrm{N} 25^{\circ} \mathrm{W}$ and a $0.1-100 \mathrm{~s}$ strike of $\mathrm{N} 20^{\circ} \mathrm{E}$. The former was associated with the allocthonous terranes in the Canadian Cordillera which are of up to $5-10 \mathrm{~km}$ thickness. The longer period strike direction, representative of the bulk of the crust below $7 \mathrm{~km}$, was interpreted as autochthonous North American basement rocks. The analysis and determination of strike variation on both is a very helpful tool for geodynamic studies. Thus, the strike variation along different profiles, determined by Ledo and Jones (2001), in the Southern Canadian Cordillera was associated with a zone of early Eocene extension in the Omineca Belt.

Simpson (2001) showed how the use of MT can resolve to some extent the ambiguity on the origin depth of seismic anisotropy in the splitting of SKS shear waves. Comparison of seismic and electrical anisotropy in the North Central craton of Australia implies that strain associated with present day plate motions have not dominated mantle deformation sufficiently to induce alignment of olivine in the direction of plate motion.

Variation of the magnetotelluric strike with depth was seen by Wu et al. (2002) in the Great Slave Lake Shear Zone (GSLsz). The analysis of the MT data showed that the geoelectric strike direction near the GSLsz varies with depth from $\mathrm{N} 30^{\circ} \mathrm{E}$ at periods less than $20 \mathrm{~s}$ (upper and middle crust) to $\mathrm{N} 60^{\circ} \mathrm{E}$ in the period range $20-1500 \mathrm{~s}$ (lower crust and upper mantle). They 

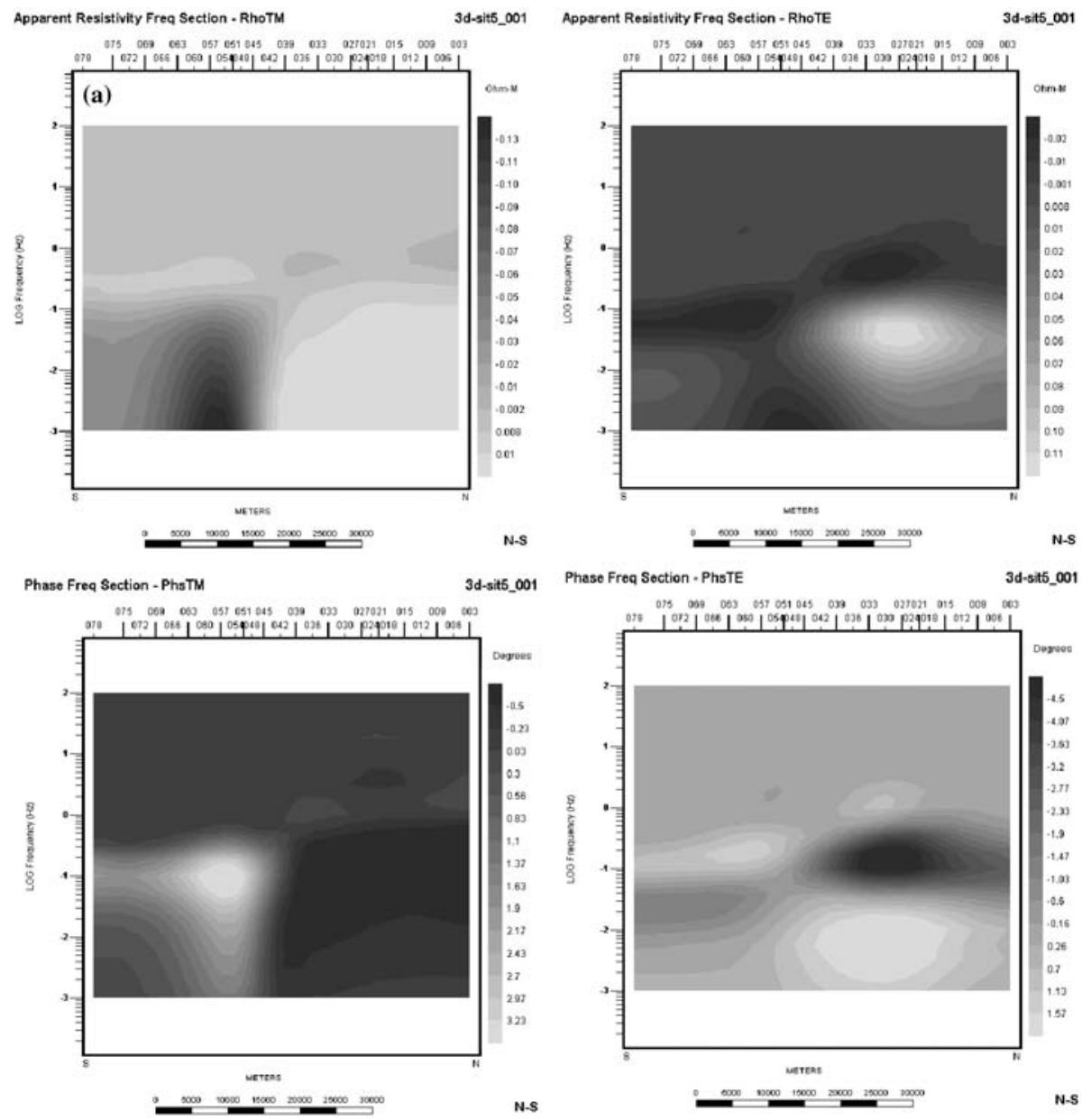

Figure 12. (a) Difference between the apparent resistivities and phases of the 2-D regional model and the quasi 2-D model for case D. Top left: apparent resistivity TM mode; Bottom right: phase TE mode; Bottom left: phase TM mode. (b) Difference between the tipper components of the 2-D regional model and the quasi 2-D model for case D. Top right: real part of transfer function in $y$-direction; Top left: imaginary part of transfer function in $y$-direction; Bottom right: real part of transfer function in $x$-direction; Bottom left: imaginary part of transfer function in $x$-direction.

obtained four different models over different period ranges and strike directions. The main result is that all of the models that they obtained provided similar geoelectric results.

Ledo et al. (2002a) have compared the results of the 2-D inversion of 3-D synthetic data showing strike-depth dependence for different strike direction, period range and modes. The inversion of the shortest period TM and TE modes data is the one that gives the smaller root mean square error (rms), 

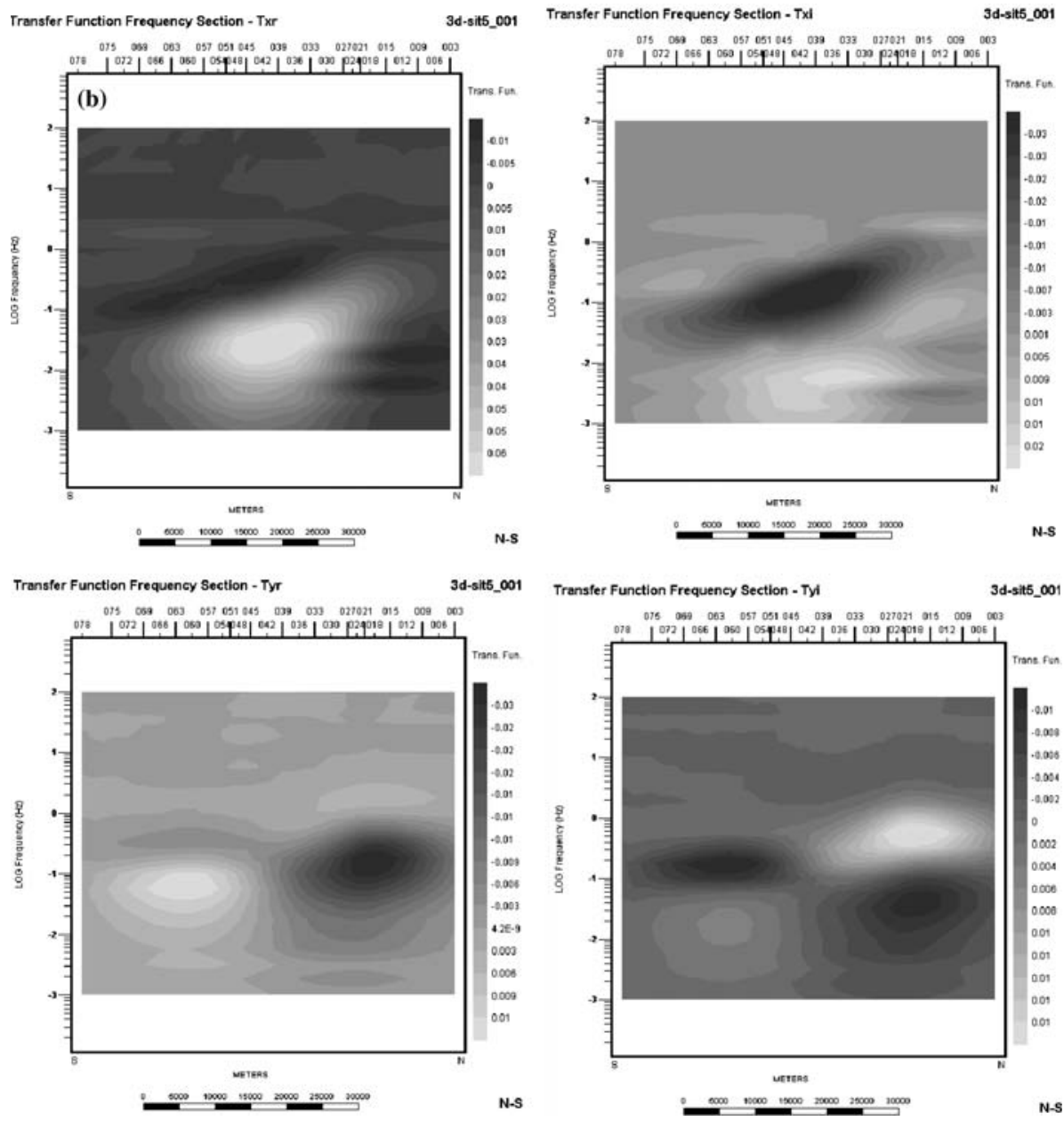

Figure 12. (continued)

and the final model obtained reproduces the main resistivity structures of the 3-D model. For the other cases, although the final models are very similar, allowing recognition of the main structures, the high final rms achieved will prevent consideration of their validity.

\section{Geophysical Aspects of 2-D Interpretation of 3-D Data}

The purpose of MT interpretation of 3-D data with 2-D techniques is to provide reliable answers to characterize the location, geometry, size, resistivity and depth of (usually) an anomalous conductor from spatial measurements; in this case, the 3-D bodies are the targets. The goal is not only to 


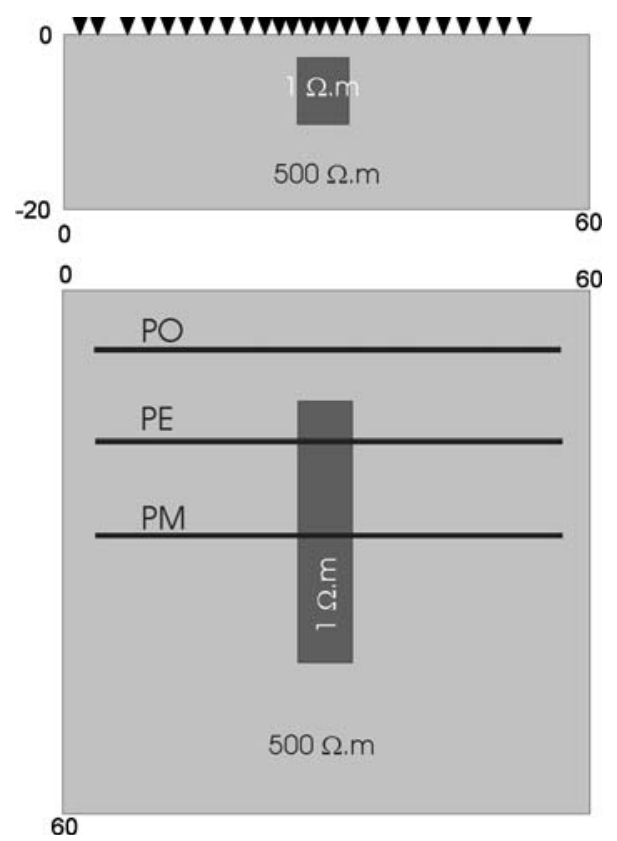

Figure 13. 3-D conductive body embedded in a homogeneous half-space. Distances are given in $\mathrm{km}$.

detect but also to delineate the 3-D structure (Queralt et al., 2002). To extract the most general results, we have considered a 3-D conductive target (Figure 13) of $1 \Omega \mathrm{m}$ embedded in a homogenous half-space of $500 \Omega \mathrm{m}$. The 3-D target horizontal dimensions are $5 \mathrm{~km} \times 30 \mathrm{~km}$ and $8 \mathrm{~km}$ thick, and its top is situated $2.5 \mathrm{~km}$ below the ground surface. In this case, we are considering an isolated target; real situations are, in general, more complicated and it will be necessary to consider the effects of other structures on the measured electromagnetic fields.

Although data acquisition on highly dense 2-D grids has been undertaken, its 3-D magnetotelluric interpretation is limited. In many cases 2-D interpretation techniques are the first step before attempting any 3-D forward modeling or inversion. We have considered three different profiles: one crossing the 3-D structure at its midpoint (PM in Figure 13), another one crossing the 3-D body close to its lateral ending (PE in Figure 13) and finally a profile located off the 3-D structure (PO in Figure 13).

Our objective is to determine which part of the 3-D dataset for each profile is more appropriate to obtain as much information as possible on the conductive target using 2-D modeling and inversion techniques. We have calculated the sensitivity matrix for a 2-D model constructed from a vertical section cutting the 3-D target. The sensitivity matrix was calculated using the 

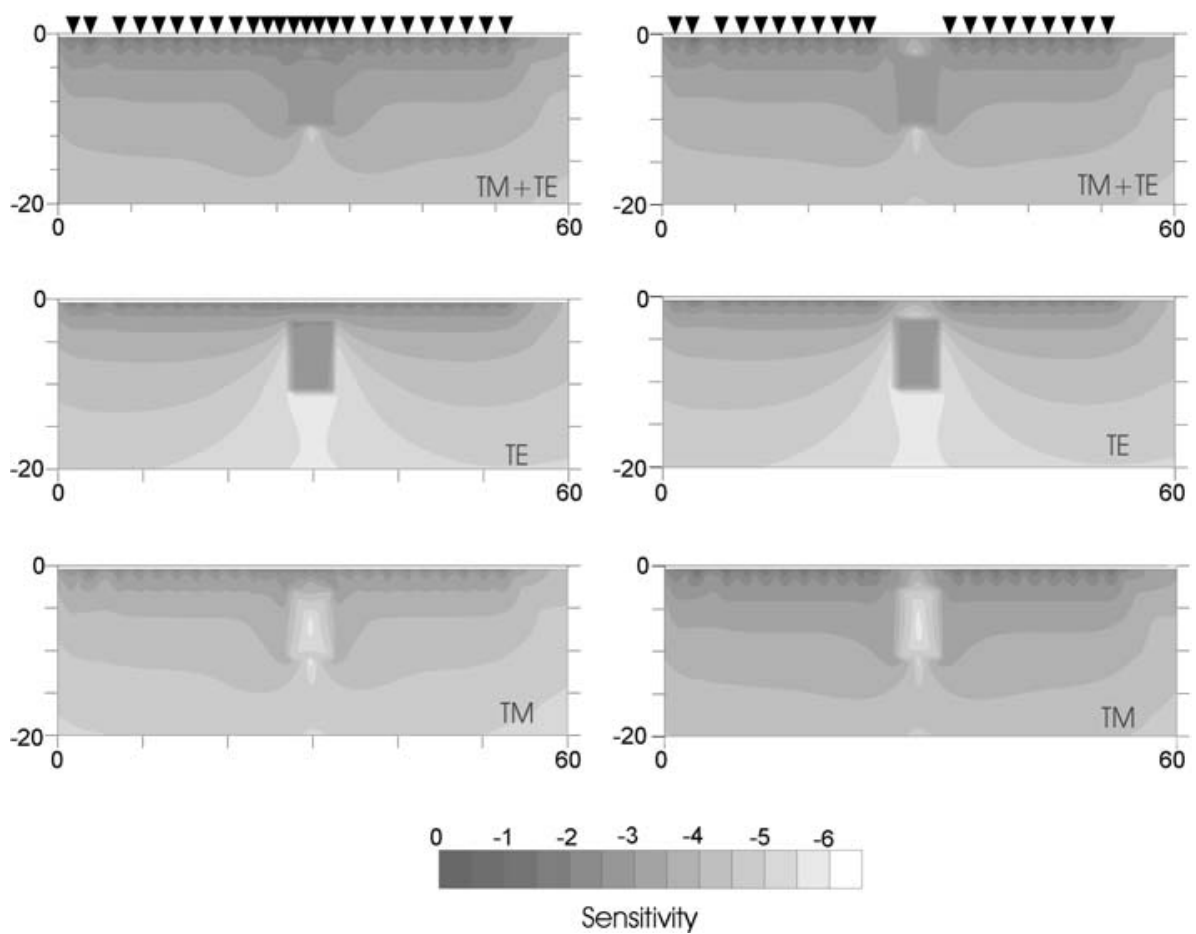

Figure 14. Contour of the normalized weighted columnwise sums of the sensitivity matrix. Vertical axe: depth in $\mathrm{km}$; horizontal axe: distance in $\mathrm{km}$.

Mackie et al. (1993) 2-D inversion code with the code modifications of Schwalenberg et al. (2002) and Brasse et al. (2002). At the same time, we have considered two different spatial distributions of the MT sites. Case A consists of a very dense station deployment and Case B consists of the same site distribution removing the sites located over the conductive target. Figure 14 show the TM, TE and joint TM + TE modes linear sensitivities for our model calculated in terms of the logarithm of the apparent resistivity and phase. It is important to note that the values obtained represent the sensitivity (or influence on the data) to small perturbations of the logarithm of resistivity in each model cell. In Case A, the use of both modes enables the delineation of the conductive target (lateral extension, top and bottom). The top and lateral extension of the body is well determined by both modes. However, the bottom of the structure can only be determined by the use of the TE mode. In case B, the results are practically identical.

We now compare the response of the 3-D model with the one obtained from the 2-D vertical section. Figure 15 illustrates the difference between the responses of both models in a pseudosection format. The difference of the TE mode presents a longer wavelength, more sites are affected, and the maxi- 

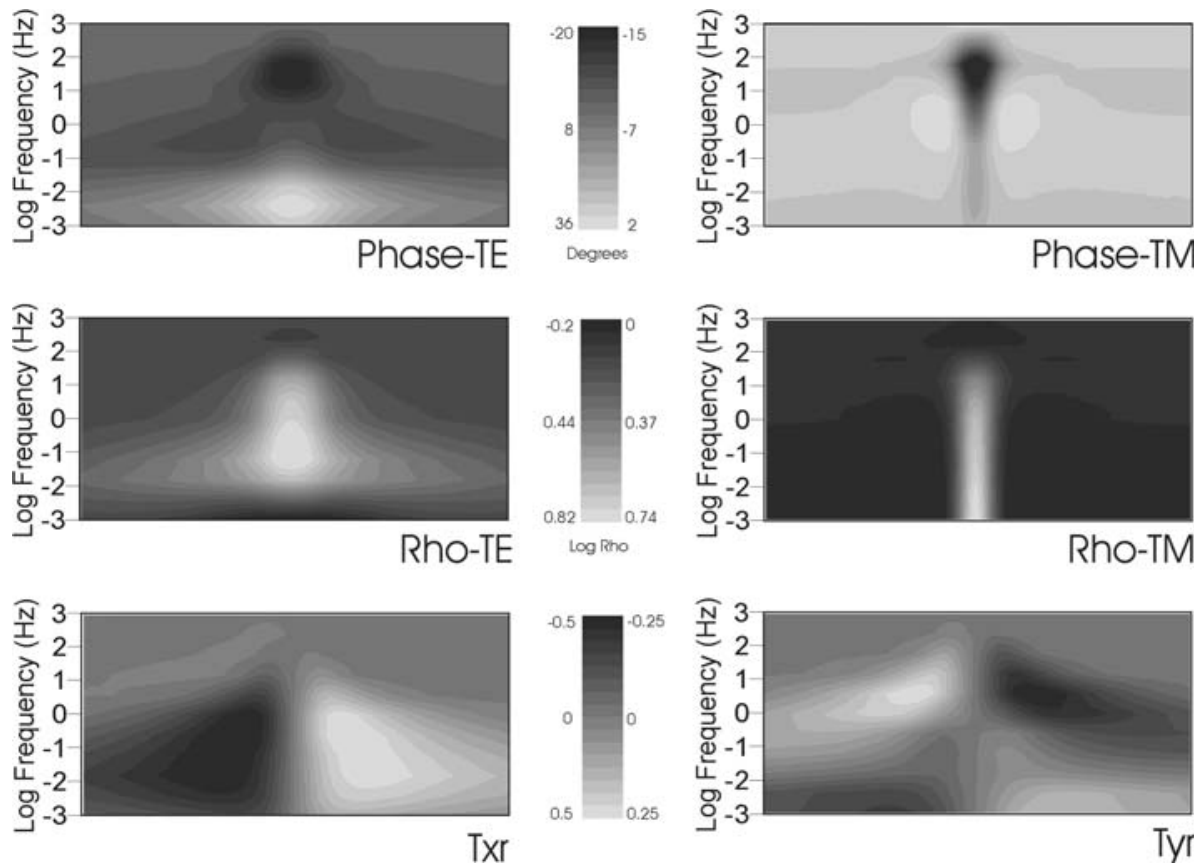

Figure 15. Difference between the 2-D regional and the 3-D data for profile PM.

mum difference corresponds to the sites located over the conductive body. The effect on the TM mode is larger for the sites located over the conductive body, while it almost vanishes for the rest. The key point of Figure 15 is the behavior of the TE mode for long periods; in the vicinity of the 3-D body, the apparent resistivities remain anomalously low and the phases are much higher compared with the 2-D response. Thus, the interpretation of these sites with 2-D techniques would considerably overestimate both the conductivity and the depth to the bottom of the 3-D structure. For the vertical magnetic component, there is also an important discrepancy between the 2-D and $3-\mathrm{D}$ response.

In the 3-D case, the values of the magnetic fields are smaller than in the 2-D case, implying that the interpretation of the vertical magnetic data with a 2-D algorithm will produce a model with a conductance substantially less than that of the true structure (Wannamaker, 1999). On the other hand, if these sites are not used (Case B), from the sensitivity test it is clear that the body can still be delineated. The TM data of the sites located over the conductive structure, although less affected than the TE mode data, are still distorted and their interpretation would underestimate the conductivity of the 3-D structure.

For an off-center profile located over the 3-D structure (PE in Figure 13) the situation is similar: a TM-only interpretation of the data will allow 

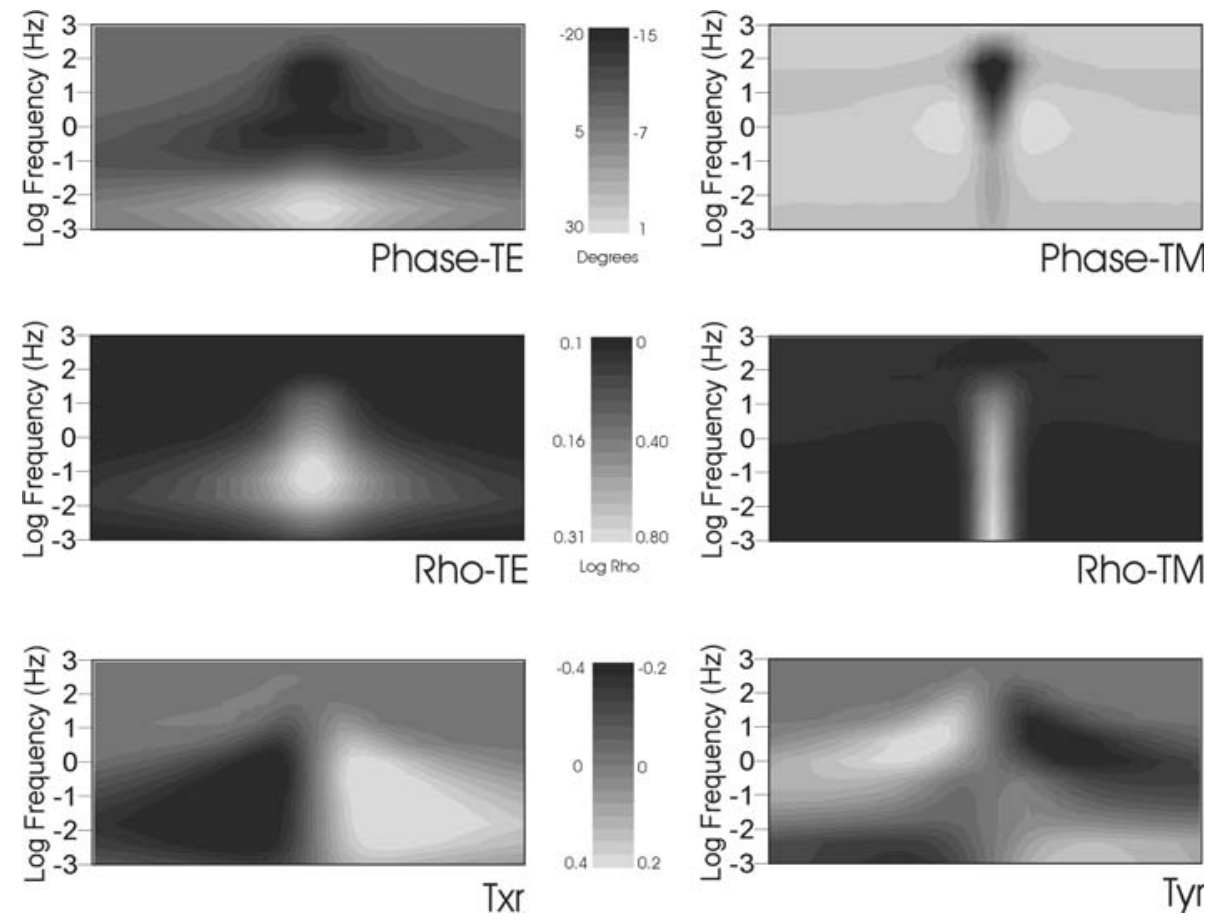

Figure 16. Difference between the 2-D regional and the 3-D data for profile PE.

determination of the lateral extension and the top of the conductive structure, but not the depth to the bottom (Figure 16). Finally, if the profile is not located over the 3-D structure (PO in Figure 13) the TE mode is again the most affected both in magnitude and in wavelength (Figure 17).

The effect of the variation of the aspect ratio for an isolated conductive 3-D body on the data acquired along a centered profile crossing the 3-D body has already been examined by Ting and Hohmann (1981). Queralt et al. (2002) presented a detailed study of the variation of the length of the long axis of a realistic 3-D ore body. They concluded that, for sites located over the ore body, the TE mode is more sensitive to the aspect ratio, than the TM mode is.

One example that shows the importance of the surrounding media to the detection and delineation of isolated ore-bodies is the MT study of the Trillabelle massive sulfide in Sudbury (Ontario, Canada) by Livelybrooks et al. (1996). 2-D inversion was used to generate 3-D models to explain the observed data. Three-dimensional forward modeling provided two classes of models that can explain some of the high phases observed. Both models suggest the presence of current gathering features. The first class involves surrounding the ore body with a partially mineralized halo, which serves to channel currents into the body. The second class connects the ore body to regional faults that feed current into the body. 

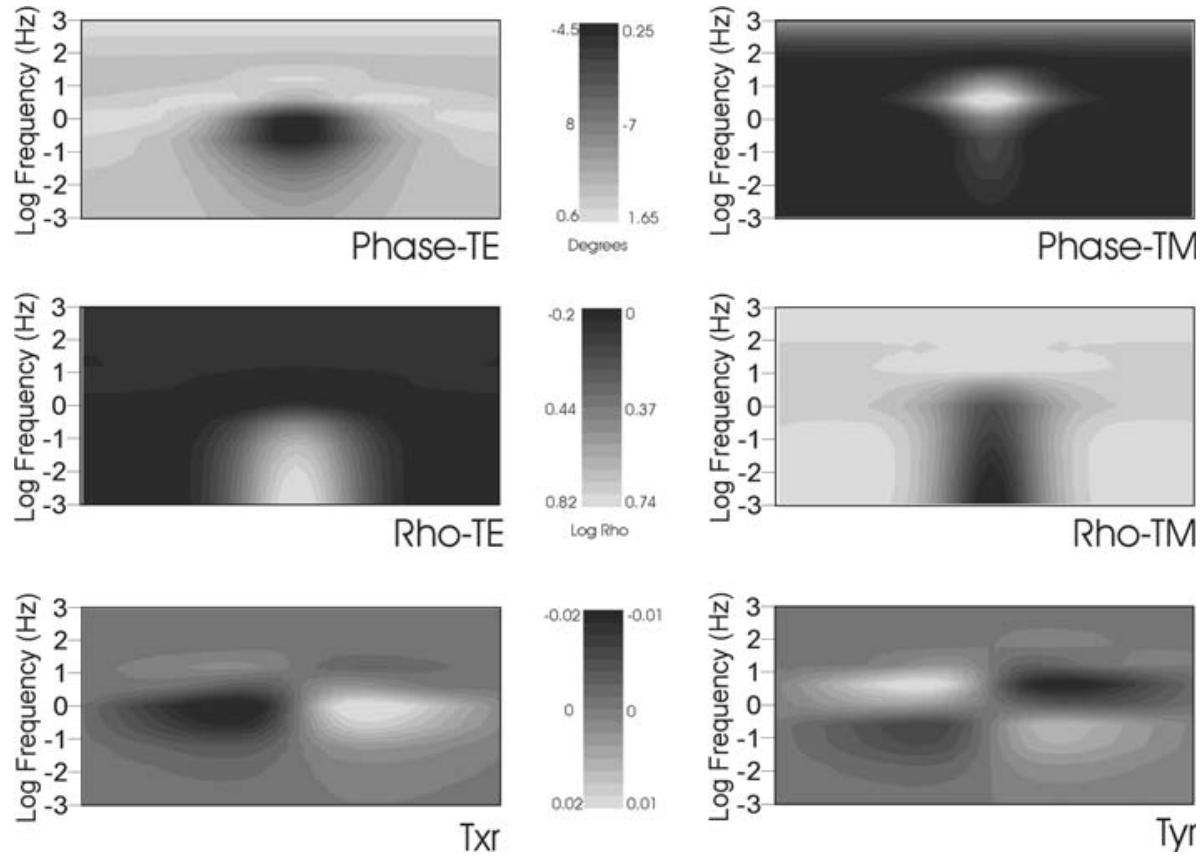

Figure 17. Difference between the 2-D regional and the 3-D data for profile PO.

\section{Discussion and Conclusions}

The application of the two-dimensionality hypothesis to the interpretation of 3-D magnetotelluric data has limits on the modes, periods and spatial ranges. The main purpose of MT interpretation of 3-D data with 2-D techniques depends on the scale of the problem. For crustal or larger scales, the goal is determination of a robust 2-D model minimally affected by the presence of medium-scale 3-D structures. For small scale problems, the main goal is providing reliable answers to characterize the location, geometry, size, resistivity and depth of (usually) a structural 3-D conductor from spatial measurements.

Due to the non-uniqueness of the magnetotelluric problem and the effect of data error, the 2-D geophysical interpretation of these data is always an iterative process. During this process, several approaches can be followed for 2-D modeling or inversion. Berdichevsky et al. (1998) and Wannamaker (1999) summarized a general scheme of 2-D MT interpretation. Following their ideas, we propose here some guidelines for potential users that can be customized to each geologic environment. The following "fuzzy" set of recommendations is based on the author's personal experience, and cannot be considered as conveying any kind of general agreement valid for all situations. 


\subsection{DATA SELECTION}

The first stage consists of combining all the geological and geophysical data available with the qualitative and quantitative analysis of the MT data to determine which subset is most suitable for interpretation. The first step is the detection and correction of distortion caused by structures of scale much smaller than those of interest. For 2-D data, application of the techniques proposed by Zhang et al. (1987), Jones (1988), Groom and Bailey (1989, 1991), Bahr (1991), Groom and Bahr (1992), Jones and Groom (1993), Chakidri et al. (1992), Chave and Smith (1994), Smith (1995), Spitzer (2001), and Ledo et al. (2002b) usually suffice. For 3-D data, the methods proposed by Ledo et al. (1998), Utada et al. (2000), and García and Jones (2002) are advocated. A complete review of different aspects and techniques can be found in the reviews by Jones (1983), Jiracek (1990) and Ogawa (2002).

The use of the impedance tensor invariant rotational parameters can also help to constrain the set of possible models, especially when there are measurements along the geological strike are available. In this case, the use of the phase of the impedance tensor determinant (Berdichevsky and Dimitriev, 1997) and other parameters defined by several authors can help to estimate the degree of horizontal inhomogeneity of the medium and help determine the dimensionality of the dataset (Vozoff, 1972; Jupp and Vozoff, 1976; Lilley, 1993; Esparza and Gomez-Treviño, 1997; Szarka and Menvielle, 1997; Makris et al., 1999; Romo et al., 1999; Weaver et al., 2000). If the geoelectrical models can be considered linear, passive and causal, then their responses are consistent with the impedance dispersion relation (Weidelt, 1972; Fischer and Schnegg, 1980; Yee and Paulson, 1988, 1990; Egbert, 1990; Weidelt and Kaikonen, 1994; Berdichevsky and Pokhotelov, 1997; Marcuello et al., 2005). The consistency between the apparent resistivities and phases can be checked with the Rho + algorithm of Parker and Booker (1996).

All of this information helps to determine the appropriate strike direction and which modes, periods and site distributions to use to initiate 2-D modeling or inversion procedures. The TM and TE modes satisfy the principle of information complementarity. The most reliable and comprehensive information on the Earth's conductivity can be obtained by means of joint TM and TE mode interpretations. The use of only part of the data (i.e. TM mode only) to avoid 3-D effects is not correct if the required conditions are not justified. Although, in magnetotellurics, Occam's razor is applied in many cases, it is important to remember that the simplest techniques are the best only if they are correct. 


\subsection{2-D INVERSION AND/OR FORWARD MODELING}

2-D inversion of selected data can be carried out with the fast and accurate 2-D inversion codes available within the MT community (i.e. Jupp and Vozoff, 1976; Constable et al., 1987; deGroot-Hedlin and Constable, 1990; Marcuello et al., 1991; Smith and Booker, 1991; Schmucker, 1993; Schnegg, 1993; Uchida, 1993; Ogawa and Uchida, 1995; Siripunvaraporn and Egbert, 2000; Rodie and Mackie, 2001). A linear sensitivity test of the final model following Schwalenberg et al. (2002) helps to find which parts of the model are determined by different modes, period ranges and sites. Examples of selective inversion can be found in Banks et al. (1996), Livelybrooks et al. (1996) and Berdichevsky et al. (1998).

Although 2-D inversion of the observed data is predominant nowadays, forward modeling, especially to carry out non-linear sensitivity tests of the main structures of the model is a highly recommended step. Along this line of reasoning, the procedure outlined by Park and Mackie (2000) to study the effects of structures parallel to the profiles using 3-D forward modeling is very helpful and straightforward. This is an iterative process, in which the initial model obtained from the inversion is examined in detail and recalculated. The final model must emphasize the most reliable -and suppress the least reliable- elements of the model derived from the different modes, periods and spatial ranges supporting a 2-D interpretation.

\subsection{Geological interpretation}

At this point, an effort to translate the electrical conductivity model (a beautiful color image for most people alien to EM methods) into geological information (i.e. tectonic, geodynamic and petrophysical meaning) must be attempted. Special attention must be put on the petrophysical interpretation of the data and its correlation with laboratory and physical studies (Duba and Shankland, 1982; Constable et al., 1992; Jones et al., 1997; Bahr and Duba, 2000) this is the kind of information which in many cases it is feasible to obtain only with EM methods (Jones, 1992). It is evident that the geological information obtained from MT data can be incorporated into the first stage of this set of recommendations.

Ting and Hohmann (1981) claimed that the magnetotelluric method was in an inferior position with respect to other geophysical methods due to a lack of interpretational capability through the inappropriate use of 1-D or 2-D interpretation techniques; 3-D codes were not readily available two decades ago. Currently, 3-D trial-and-error forward codes are available and 3-D inversions are emerging. However, even for a high density grid of stations and efficient 3-D inversion codes, there will always be the problem of spatial aliasing. We cannot model the Earth at all scales from the sub-elec- 
trode array scale (meters) to the lithospheric scale (tens to hundreds of kilometers), so there may sometimes be a need for pre-processing of the measured responses to remove the effects of small-scale scatterers. Often a 2-D inversion of data that exhibit weak 3-D effects is undertaken because of the inadequacy of spatial coverage (e.g., only a single profile of data, rather than a grid), or because of the complexity of 3-D modeling.

Thus, studies of the effects of 3-D structures in a 2-D inversion are necessary to ascertain when a 2-D approximation may be valid. 2-D techniques applied to 3-D data are often considered an adequate approach to answer simple "yes or no" geological questions, but can be misleading if used without thought, as simple questions do not necessarily have trivial answers. A clear example of the limitation of 2-D interpretation of 3-D data was presented by García et al. (1999) for the Kayabe dataset. These authors showed that, although 2-D models were consistent with 'TM' and 'TE' quantities, when a 3-D model was created using the 2-D models there was an important misfit between the data and the 3-D model responses. Moreover, as Park and Torres-Verdin (1988) stated, we must remember that "3-D modeling simply cannot be avoided in complex geological environments".

\section{Acknowledgements}

The author thanks the IAGA working group 1.2 for giving him the opportunity to present a review of this topic. He acknowledges all the people who replied to the e-mail asking for comments and references of interest for this work; Katrin Schwalenberg kindly provided her scripts to perform the linear sensitivity analysis of 2-D models. The author has benefited enormously from the scientific comments of, and discussions with, several colleagues from the Geological Survey of Canada and from the Universitat de Barcelona. In particular, he owes special thanks to Alan G. Jones, Pilar Queralt and Alex Marcuello, not only for sharing their scientific views and thoughts but also for their encouragement. Finally, he thanks Alan Chave and George Jiraceck and two anonymous reviewers for their substantial comments on the original version of this manuscript. The author thanks Michael Rycroft, Editor-inChief of Surveys in Geophysics, for his suggestions.

\section{Appendix} $\mathbf{A}=\frac{1}{4 \pi} \iiint_{V} \frac{\mathrm{e}^{-i k\left|\mathbf{r}-\mathbf{r}^{\prime}\right|}}{\left|\mathbf{r}-{ }^{-}\right|} d V$ is the vector potential solution of the Helmholtz equa-
tion

$$
\nabla^{2}+\mathbf{A}+k^{2} \mathbf{A}=-\mathbf{J}
$$


with $\mathbf{J}$ being the source current density and $k^{2}=i \omega \mu \sigma_{\text {body }}$ The electric field can be expressed as:

$$
\begin{aligned}
& \overrightarrow{E^{\Theta}}=-i \omega \mu \frac{1}{4 \pi} \iiint_{V} \frac{\mathbf{J e}^{-i k\left|\mathbf{r}-\mathbf{r}^{\prime}\right|}}{\left|\mathbf{r}-\mathbf{r}^{\prime}\right|} d V+\frac{1}{i \omega \varepsilon} \nabla\left(\nabla \cdot \frac{1}{4 \pi} \iiint_{V} \frac{\mathbf{J} \mathrm{e}^{-i k\left|\mathbf{r}-\mathbf{r}^{\prime}\right|}}{\left|\mathbf{r}-\mathbf{r}^{\prime}\right|} d V\right) \\
&=-i \omega \mu \frac{1}{4 \pi} \iiint_{V} \frac{\mathbf{J} \mathrm{e}^{-i k\left|\mathbf{r}-\mathbf{r}^{\prime}\right|}}{\left|\mathbf{r}-\mathbf{r}^{\prime}\right|} d V+\frac{1}{i \omega \varepsilon} \nabla\left(\frac{1}{4 \pi} \iiint_{V} \nabla \cdot\left(\frac{\mathbf{J} \mathrm{e}^{-i k\left|\mathbf{r}-\mathbf{r}^{\prime}\right|}}{\left|\mathbf{r}-\mathbf{r}^{\prime}\right|}\right) d V\right. \\
&=-i \omega \mu \frac{1}{4 \pi} \iiint_{V} \frac{\mathbf{J} \mathrm{e}^{-i k\left|\mathbf{r}-\mathbf{r}^{\prime}\right|}}{\left|\mathbf{r}-\mathbf{r}^{\prime}\right|} d V+\frac{1}{i \omega \varepsilon} \nabla\left(\frac{1}{4 \pi} \iint_{\mathrm{S}} \nabla\left(\frac{\mathbf{J} \mathrm{e}^{-i k\left|\mathbf{r}-\mathbf{r}^{\prime}\right|}}{\left|\mathbf{r}-\mathbf{r}^{\prime}\right|}\right) d S\right)
\end{aligned}
$$

The first term of the right side of the equation represents the volume currents within the body (induction term) while the second term represents charge accumulation at the conductivity discontinuities. The magnetic field can be expressed as:

$$
\mathbf{H}^{\mathrm{s}}=-\nabla \mathbf{A}=\frac{1}{4 \pi} \iiint_{V} \frac{\mathbf{J} \mathrm{e}^{-i k\left|\mathbf{r}-\mathbf{r}^{\prime}\right|}}{\left|\mathbf{r}-\mathbf{r}^{\prime}\right|} d V
$$

\section{References}

Ádám, A., and Kis, M.: 2001. 'Phase Paradox in the Bekes Basin and a Possibility for its Resolution by 2D/3D Modeling', Acta Geod. Geoph. Hung. 36, 133-152.

Alumbaugh, D. L., and Newman, G. A.: 2000. 'Image Appraisal for 2-D and 3-D Electromagnetic Inversion', Geophysics 65, 1455-1467.

Banks, R. J., Livelybrooks, D., Jones, P., and Longstaff, R.: 1996. 'Causes of High Crustal Conductivity Beneath the Iapetus Suture Zone in Great Britain', Geophys. J. Int. 124, 433-455.

Bahr, K.: 1991. 'Geological Noise in Magnetotelluric Data: A Classification of Distortion Types', Phys. Earth Planetary Interiors 66, 24-38.

Bahr, K., and Duba, A.: 2000. 'Is the Asthenosphere Electrically Anisotropic?', Earth Planet. Sci. Lett. 178, 87-95.

Berdichevsky, M. N., and Dmitriev, V. I.: 1997. 'On Deterministic Nature of Magnetotelluric Impedance', Acta Geophysica Polonica, XLV 3, 227-237.

Berdichevsky, M. N., and Pokhotelov, D. O.: 1997. 'Violation of the Dispersion Relations in a Three-Dimensional Magnetotelluric Model', Izvestiya, Phys. Solid Earth 33, 603-608.

Berdichevsky, M. N., Dmitriev, V. I., and Pozdnjakova, E. E.: 1998. 'On Two-Dimensional Interpretation of Magnetotelluric Soundings', Geophys. J. Int. 133, 585-606.

Berdichevsky, M. N.: 1999. 'Marginal Notes on Magnetotellurics', Surveys Geophys. 20, 341-375.

Brasse H., Lezaeta P., Rath V., Schwalenberg K., Soyer W. and Haak V.: 2002, 'The Bolivian altiplano conductivity anomaly'. J. Geophys. Res., 107 (B5). 10.1029/2001JB000391. 
Chakridi, R., Chouteau, M., and Mareschal, M.: 1992. 'A Simple Technique for Analysing and Partly Removing Galvanic Distortion from the Magnetotelluric Impedance Tensor: Application to Abitibi and Kapuskasing data (Canada)', Geophys. J. Int. 108, 917-929.

Chave, A. D., and Smith, J. T.: 1994. 'On electric and Magnetic Galvanic Distortion Tensor Decompositions', J. Geophys. Res. 99, 4669-4682.

Constable, S. C., Parker, R. L., and Constable, C. G.: 1987. 'Occam's Inversion: A Practical Algorithm for Generating Smooth Models from Electromagnetic Sounding Data', Geophysics 52, 289-300 .

Constable, S., Shankland, T. J., and Duba, A.: 1992. 'The Electrical Conductivity of an Isotropic Olivine Mantle', J. Geophys. Res. 97, 3397-3404.

Dosso, H. W., and Chen, J.: 2000. 'Analogue Model Study of EM Induction in Elongated Conductors - 2D and 3D Induction Arrow Responses', Earth Planets Space 52, 355-360.

Duba, A. L., and Shankland, T. J.: 1982. 'Free Carbon and Electrical Conductivity in the Earth's Mantle', Geophys. Res. Lett. 9, 1271-1274.

Egbert, G.: 1990. 'Comments on Concerning Dispersion Relations for the Magnetotelluric Impedance Tensor by E. Yee and K.V. Paulson', Geophys. J. Int. 102, 1-8.

Esparza, F. J., and Gomez-Treviño, E.: 1997. 'Discussion on: "The Magnetotelluric Method in the Exploration of Sedimentary Basins" Vozoff, K., with reply by the authors', Geophysics 62, 691-692.

Fischer, G., and Schnegg, P. A.: 1980. 'The Dispersion Relations of the Magnetotellutic Response and their Incidence on the Inverse Problem', Geophys. J. Roy. Astron. Soc. 62, 661-673.

García, X., Ledo, J., and Queralt, P.: 1999. '2-D Inversion of 3-D Magnetotelluric Data: The Kayabe Dataset', Earth, Planets Space 51, 1135-1143.

García, X., and Jones, A. G.: 2003. 'Extended Decomposition of MT Data', in M. S. . Zhdanov, and P. E. Wannamaker (eds.), Three-Dimensional Electromagnetics-II, Second International Gerald W. Hohmann Symposium, Elsevier, Amsterdam.

Groom, R. W., and Bailey, R. C.: 1989. 'Decomposition of the Magnetotelluric Impedance Tensor in the Presence of Local Three-Dimensional Galvanic Distortion', J. Geophys. Res. 94, 1913-1925.

Groom, R. W., and Bailey, R. C.: 1991. 'Analytical Investigations of the Effects of NearSurface Three-Dimensional Galvanic Scatterers on MT Tensor Decomposition', Geophysics 56, 496-518.

Groom, R. W., and Bahr, K.: 1992. 'Corrections for Near Surface Effects: Decomposition of the Magnetotelluric Impedance Tensor and Scaling Corrections for Regional Resistivities: A Tutorial', Surveys Geophys. 13, 341-380.

de Groot-Hedlin, C., and Constable, S.: 1990. 'Occam's Inversion to Generate Smooth Two-Dimensional Models from Magnetotelluric Data', Geophysics 55, 1613-1624.

Habashy, T. M., Groom, R. G., and Spies, B. R.: 1993. 'Beyond the Born and Rytov Approximations: A Nonlinear Approach to Electromagnetic Scattering', J. Geophys. Res. 98, 1759-1775.

Harrington, R. F.: 1961, Time-Harmonic Electromagnetic Fields, McGraw-Hill, New York.

Hermance, J. F.: 1982. 'The Asymptotic Response of Three-Dimensional Basin Offsets to Magnetotelluric Fields at Long Periods: The Effect of Current Channeling', Geophysics 47(11), 1562-1573.

Hoffmann-Rothe, A., Ritter, O., and Haak, V.: 2001. 'Magnetotelluric and Geomagnetic Modelling Reveals Zones of Very High Electrical Conductivity in the Upper Crust of Central Java', Phys. Earth Planetary Interiors 124, 131-151.

Hohmann, G. W.: 1975. 'Three-Dimensional Induced Polarization and Electromagnetic Modelling', Geophysics 40, 309-324. 
Jackson, J. D.: 1975, Classical Electrodynamics, John Wiley \& Sons, New York.

Jiraceck, G.: 1990. 'Near-Surface and Topographic Distortions in Electromagnetic Induction', Surveys Geophys. 11, 163-203.

Jones, A. G.: 1983. 'The Problem of Current Channeling: A Critical Review', Geophys. Surveys 6, 79-122.

Jones, A. G.: 1988. 'Static Shift of Magnetotelluric Data and its Removal in a Sedimentary Basin Environment', Geophysics 53, 967-978.

Jones, A. G.: 1992. 'Electrical Conductivity of the Continental Lower Crust', in D. M. Fountain, R. J. Arculus, and R. W. Kay (eds.), Continental Lower Crust, Elsevier, Amsterdam, pp. 81-143, Chapter 3.

Jones, A. G., and Groom, R. W.: 1993. 'Strike Angle Determination from the Magnetotelluric Tensor in the Presence of Noise and Local Distortion: Rotate at Your Peril!', Geophys. J. Int. 113, 524-534.

Jones, A. G., Katsube, T. J., and Schwann, P.: 1997. 'The Longest Conductivity Anomaly in the World Explained: Sulphides in Fold Hinges Causing Very High Electrical Anisotropy', J. Geomag. Geoelec. 49, 1619-1629.

Jones, F. W., and Vozoff, K.: 1978. 'The Calculation of Magnetotelluric Quantities for ThreeDimensional Conductivity Inhomogeneities', Geophysics 43, 1167-1175.

Jupp, D. L. B., and Vozoff, K.: 1976. 'Two-Dimensional Magnetotelluric Inversion', Geophys. J. Roy. Astron. Soc. 50, 333-352.

Kaufman, A. A., and Keller, G. V.: 1981, The magnetotelluric sounding method, In methods in Geochemistry and Geophysics, Elsevier, Amsterdam.

Ledo, J., Queralt, P., and Pous, J.: 1998. 'Galvanic Distortion on Magnetotelluric Data over a 3D Regional Structure', Geophys. J. Int. 132, 295-301.

Ledo, J, Ayala, C, Pous, J, Queralt, P, Marcuello, A., and Muñoz, J. A.: 2000. 'New Geophysical Constraints on the Deep Structure of the Pyrenees', Geophys. Res. Lett. 27, 1037-1040.

Ledo J. and Jones A.G.: 2001, 'Regional electrical resistivity structure of the southern Canadian Cordillera and its physical interpretation'. J.Geophys. Res. 106, 30,755-30,770.

Ledo, J, Queralt, P, Martí, A., and Jones, A. G.: 2002a. 'Two-Dimensional Interpretation of Three-Dimensional Magnetotelluric Data: an Example of Limitations and Resolution', Geophys. J. Int. 150, 127-139.

Ledo, J, Gabás, A., and Marcuello, A.: 2002b. 'Static Shift Levelling using Geomagnetic Transfer Functions', Earth, Planets Space 54, 493-498.

Lilley, F. E. M.: 1993. 'Magnetotelluric Analysis using Mohr Circles', Geophysics 58, 14981506.

Livelybrooks, D, Mareschal, M, Blais, E., and Smith, J. T.: 1996. 'Magnetotelluric Delineation of the Trillabelle Massive Sulfide Body in Sudbury, Ontario', Geophysics 61, 971-986.

de Lugao P.P. and Kriegshauser B.: 1997, '2-D inversion of magnetotelluric data over 3-D structures', 67th Ann. Internat. Mtg. Soc. of Expl. Geophys. 382-385.

Mackie, R. L., and Madden, T. R.: 1993. 'Three-Dimensional Magnetotelluric Inversion using Conjugate Gradients', Geophys. J. Int. 115(1), 215-229.

Mackie, R. L., Smith, T. J., and Madden, T. R.: 1994. 'Three-Dimensional Electromagnetic Modeling using Finite Differences Equations: The Magnetotelluric Example', Radio Sci. 29, 923-935.

Makris, J, Bogris, N., and Eftaxias, K.: 1999. 'A New Approach in the Determination of Characteristic Directions of the Geoelectric Structure using Mohr Circles', Earth, Planets Space 51, 1059-1065. 
Marcuello, A., Kaikkonen, P., and Pous, J.: 1991. '2-D Inversion of Mt Data with a Variable Model Geometry', Geophys. J. Int. 110, 297-394.

Marcuello A., Queralt P. and Ledo J.: 2005, 'The use of Dispersion Relations for the Geomagnetic Transfer Functions', Phys. Earth and Planetary Interiors, doi:10.1016/ j.pepi.2004.08.016, 150, 85-91.

Marquis, G., Jones, A. G., and Hyndman, R. D.: 1995. 'Coincident Conductive and Reflective Lower Crust Across a Thermal Boundary in Southern British Columbia, Canada', Geophys. J. Int. 120, 111-131.

Monteiro Santos, F. A., Matos, L., Almeida, E., Matias, H., Mateus, A., and Mendes-Victor, L. A.: 2002. 'Three-Dimensional Magnetotelluric Modelling of the Vilariça Depression (NE Portugal)', J. Appl. Geophys. 49, 59-74.

Newman, G. A., and Alumbaugh, D. L.: 2000. 'Three-Dimensional Magnetotelluric Inversion using Non-Linear Conjugate Gradients', Geophys. J. Int. 140(2), 410-424.

Newman, G. A. Hoversten, G. M. and Alumbaugh, D. L.: 2002, 'Three dimensional magnetotelluric modeling and inversion: application to sub-salt imaging' in Methods in Geochemistry and Geophysics, 35: Three-Dimensional Electromagnetics: Proceedings of the Second International Symposium (Methods in Geochemistry and Geophysics. Eds: M. S. Zhdanov and P. E. W. Wannamaker, 127-152.

Ogawa, Y., and Uchida, T.: 1995. 'A Two-Dimensional Magnetotelluric Inversion with Assuming Gaussian Static Shift', Geophys. J. Int. 126, 69-76.

Ogawa, Y.: 2002. 'On Two-Dimensional Modeling of Magnetotelluric Field Data', Surveys Geophys. 23, 251-272.

Osella, A., Martinelli, P. and Tortorella, D.: 2000, Detection of 3D conductive structures by 2D inversion of MT data. 15th Workshop on Electromagnetic Induction in the Earth. Cabo Frio, Brazil.

Park, S. K.: 1985. 'Distortion of Magnetotelluric Sounding Curves by Three-Dimensional Structures', Geophysics 50, 785-797.

Park, S. K., Orange, A. S., and Madden, T. R.: 1983. 'Effects of Three-Dimensional Structure on Magnetotelluric Sounding Curves', Geophysics 48, 1402-1405.

Park, S. K., and Torres-Verdin, C.: 1988. 'A Sytematic Approach to the Interpretation of Magnetotelluric Data in Volcanic Environments with Applications to the Quest for Magma in Long Valley, California', J. Geophys. Res. 93, 13265-13283.

Park, S. K., and Mackie, R. L.: 1997. 'Crustal Structure at Nanga Parbat, Northern Pakistan, from Magnetotelluric Soundings', Geophys. Res. Lett. 24, 2415-2418.

Park, S. K., and Mackie, R. L.: 2000. 'Resistive (dry?) Lower Crust in an Active Orogen, Nanga Parbat, Northern Pakistan', Tectonophysics 316, 359-380.

Parker, R. L., and Booker, J. R.: 1996. 'Optimal One-Dimensional Inversion and Bounding of Magnetotelluric Apparent Resistivity and Phase Measurements', Phys. Earth Planetary Interiors 98, 269-282.

Queralt, P., Jones, A. G., Ledo, J.: 2002, 'Deep Electromagnetic Imaging of the Bathurst No. 12 Deposit: 3-D forward modelling, 2-D inversion and sensitivity tests'. Current Research, 2002-D3.

Rodi, W., and Mackie, R. L.: 2001. 'Nonlinear Conjugate Gradients Algorithm for 2-D Magnetotelluric Inversion', Geophysics 66, 174-187.

Romo, J. M., Gómez-Treviño, ., and Esparza, F. J.: 1999. 'An Invariant Representation for the Magnetic Transfer Function in Magnetotellurics', Geophysics 64, 1418-1429.

Sasaki, Y.: 2001. 'Full 3-D Inversion of Electromagnetic Data on PC', J. Appl. Geophys. 46(1), $45-54$.

Schmucker, U.: 1993. '2D Modeling with Linearized Integral Equations', J. Geomag. Geoelec. 45, 1045-1062. 
Schnegg, P. A.: 1993. 'An Automatic Scheme for 2-D Modelling, based on Low-Order Polynomial Fitting', J. Geomag. Geoelec. 45, 1039-1043.

Schwalenberg, K., Rath, V., and Haak, V.: 2002. 'Sensitivity Studies Applied to a 2-D Resistivity Model for the Central Andes', Geophys. J. Int. 150, 673-686.

Simpson, F.: 2000. 'A Three-Dimensional Electromagnetic Model of the Southern Kenya Rift: Departure from Two-Dimensionality as a Possible Consequence of a Rotating Stress Field', J. Geophys. Res. 105, 19321-19344.

Simpson, F.: 2001. 'Resistance to Mantle Flow Inferred from the Electromagnetic Strike of the Australian Upper Plate', Nature 412, 632-634.

Siripunvaraporn, W., and Egbert, G.: 2000. 'An Efficient Data-Subspace Inversion Method for 2-D Magnetotelluric Data', Geophysics 65(3), 791-803.

Siripunvaraporn, W., Egbert, G., and Uyeshima, M.: 2005. 'Interpretation of two-dimensional inversion: synthetic examples', Geophys. J. Int. 160, 804-814.

Smith, J. T.: 1995. 'Understanding Telluric Distortion Matrices', Geophys. J. Int. 122, 219-226.

Smith, J. T., and Booker, J. R.: 1991. 'Rapid Inversion of Two and Three-Dimensional Magnetotelluric Data', J. Geophys. Res. 96, 3905-3922.

Spichak, V. V., Menvielle, M., and Roussignol, M.: 1999. 'Three-Dimensional Inversion of the Magnetotelluric Fields Using Bayesian Statistics', in M. Oristaglio, and B.. Spies (eds.), 3-D Electromagnetics, SEG Publ. (GD7), Tulsa, USA, pp. 406-417.

Spichak, V. V.: 1999. 'Imaging Volcanic Interiors with MT Data', in M. Oristaglio, and B. Spies (eds.), 3-D Electromagnetics, SEG Publ. (GD7), Tulsa, USA, pp. 418-425.

Spitzer, K.: 2001. 'Magnetotelluric Static Shift and Direct Current Sensitivity', Geophys. J. Int. 144, 289-299.

Szarka, L., Menvielle, M., Tarits, P., and Ádám, A.: 1994. 'A Thin Sheet Numerical Study of the Electromagnetic Field over Geometrically Complex High Conductivity Structures: On the Current Channeling in High Conductivity 3-D Models', Acta Geod. Geoph. Hungarica 29, 125-138.

Szarka, L., and Menvielle, M.: 1997. 'Analysis of Rotational Invariants of the Magnetotelluric Impedance Tensor', Geophys. J. Int. 129, 133-142.

Takasugi, S., Tanaka, K., Kawakami, N., and Muramatsu, S.: 1992. 'High Spatial Resolution of the Resistivity Structure Revealed by a Dense Network MT Measurements - A Case Study in the Minabikayabe area, Hokkaido, Japan', J. Geomag. Geoelec. 44, 289-308.

Ting, S. C., and Hohmann, G. W.: 1981. 'Integral Equation Modelling of Three-Dimensional Magnetotelluric Response', Geophysics 46, 182-197.

Tournerie, B., Chouteau, M., Fox, L., and Nagy, Z.: 2000, Three dimensional magnetotelluric survey in Hungary, SEG Annual Meeting, Calgary.

Uchida, T.: 1993. '2-D Inversion of Coprod2 Magnetotelluric Data by use of ABIC Minimization Method', J. Geomag. Geoelec. 45, 1063-1071.

Unsworth, M. J., Egbert, G., and Booker, J. R.: 1999. 'High Resolution Electromagnetic Imaging of the San Andreas Fault in Central California', J. Geophys. Res. 104, 1131-1150.

Utada, H., and Munekane, H.: 2000. 'On galvanic Distortion of Regional Three-Dimensional Magnetotellurics Impedances', Geophys. J. Int. 140, 385-398.

Vozoff, K.: 1972. 'The Magnetotelluric Method in the Exploration of Sedimentary Basins', Geophysics 37, 98-141.

Wannamaker, P. E., Hohmann, G. W., and San Filipo, W. A.: 1984a. 'Electromagnetic Modelling of Three-Dimensional Bodies in Layered Earths using Integral Equations', Geophysics 49, 60-74.

Wannamaker, P. E., Hohmann, G. W., and Ward, S. H.: 1984b. 'Magnetotelluric Responses of Three-Dimensional Bodies in Layered Earths', Geophysics 49, 1517-1533. 
Wannamaker, P. E., Wright, P. M., Zhou, Z. X., Li, X. B., and Zhao, J. X.: 1991. 'Magnetotelluric Transect of Long Valley Caldera: Resistivity Cross Section, Structural Implications, and the Limitsof a Two-Dimensional Analysis', Geophysics 56, 926-949.

Wannamaker P. E.: 1999, 'Affordable Magnetotellurics: Interpretation in Natural Environments', In Three-dimensional Electromagnetics, Eds. Michael Oristaglio and Brian Spies. Geophysical development series v. 7. Society of Exploration Geophysicists. 349-379.

Weaver, J. T., Agarwal, A. K., and Lilley, F. E. M.: 2000. 'Characterization of the Magnetotelluric Tensor in Terms of its Invariants', Geophys. J. Int. 141, 321-336.

Weidelt, P.: 1972. 'The Inverse Problem of Geomagnetic Induction', Zeitschrift für Geophysik 8, 257-290.

Weidelt, P.: 1975. 'Electromagnetic Induction in Three-Dimensional Structures', J. Geophys. 41, 85-109.

Weidelt, P., and Kaikkonen, P.: 1994. 'Local 1-D Interpretation of Magnetotelluric B-Polarization Impedances', Geophys. J. Int. 117, 733-748.

Wu, X., Ferguson, I. J., and Jones, A. G.: 2002. 'Magnetotelluric Response and Geoelectric Structure of Great Slave Lake Shear Zone Along Lithoprobe SNORCLE Corridor 1A', Earth Planet. Sci. Lett. 196, 35-50.

Yee, E., and Paulson, K. V.: 1988. 'Concerning Dispersion Relations for the Magnetotelluric Impedance Tensor', Geophys. J. Int. 95, 549-559.

Yee, E., and Paulson, K. V.: 1990. 'Replay to the Comments on Concerning Dispersion Relations for the Magnetotelluric Impedance Tensor by G Egbert', Geophys. J. Int. 102, 9-13.

Zhang, P., Roberts, R. G., and Pedersen, L. B.: 1987. 'Magnetotelluric Strike Rules', Geophysics 52, 267-278.

Zhang, P., King, A. and Watts, D.: 1998, 'Using magnetotellurics for mineral exploration', SEG Annual Meeting Expanded Technical Program Abstracts with Biographies, 68.

Zhdanov, M. S., Fang, S., and Hursan, G.: 2000. 'Electromagnetic Inversion using Quasi-Linear Approximation', Geophysics 65, 1501-1513. 